\title{
TRANSMISSIVE BEAM STEERING THROUGH ELECTROWETTING MICROPRISM ARRAYS
}

\author{
Thesis \\ Submitted to \\ the School of Engineering of the \\ UNIVERSITY OF DAYTON
}

In Partial Fulfillment of the Requirements for

The Degree of

Master of Science in Electro-Optics

\author{
By \\ Wei Han \\ UNIVERSITY OF DAYTON \\ Dayton, Ohio
}

December, 2009 


\section{TRANSMISSIVE BEAM STEERING THROUGH ELECTROWETTING}

\section{MICROPRISM ARRAYS}

APPROVED BY:

Joseph W. Haus, Ph.D.

Advisor

Professor and Director

Electro-Optics Graduate Program
Qiwen Zhan, Ph.D.

Committee Member

Associate Professor

Electro-Optics Graduate Program

Andrew M. Sarangan, Ph.D.

Committee Member

Associate Professor

Electro-Optics Graduate Program

Malcolm W. Daniels, Ph.D.

Tony E. Saliba, Ph.D.

Associate Dean

Dean, School of Engineering

School of Engineering 
C Copyright by

Wei Han

All rights reserved

2009 


\section{ABSTRACT \\ TRANSMISSIVE BEAM STEERING THROUGH ELECTROWETTING MICROPRISM ARRAYS}

Name: Han, Wei

University of Dayton

Advisor: Dr. Joseph W. Haus

In this study, the electrowetting phenomenon is reviewed and two types of Electrowetting Microprism (EMP) beam steering devices are proposed and theoretically characterized: single-prism and bi-prism EMP arrays. For this thesis, an extended beam propagation method is used and numerical calculations of near-field and far-field intensities are performed for both designs. I investigated both one-dimensional and two-dimensional versions of the EMP arrays and determine the beam steering efficiency for both 1D and 2D cases using an incident Gaussian beam. For the phased arrays the diffraction angles are discrete due to the well known grating effect. The angles between the diffraction peaks can be covered by applying a tilted phase to the input field. The efficiency is measured over continuously changed far-field angles for different situations. 


\section{ACKNOWLEDGEMENTS}

I would like to give my deepest thanks to Dr. Joseph W. Haus, my advisor, for providing the time, ideas and everything that is required for the work reported herein, and for directing publications related to this work and this thesis and bringing them to their final conclusion with patience and expertise.

I also would like to thank all those who have given me the opportunities to fulfill the thesis work. I want to express my thanks to Dr. Jason Heikenfeld and his group for proposing such a good idea and fabricating the device. I also want to express my appreciation to all the professors in Electro-Optics Program. I would not accomplish this without their guidance and help. I also want to express my thanks to Dr. John Loomis and Prof. Charles Gauder for their support in my teaching assistantship work.

Especially, I would like to thank my parents, Jie Han and Zhongping Wang, as well as Wen Cheng. They are always there for me. 


\section{TABLE OF CONTENTS}

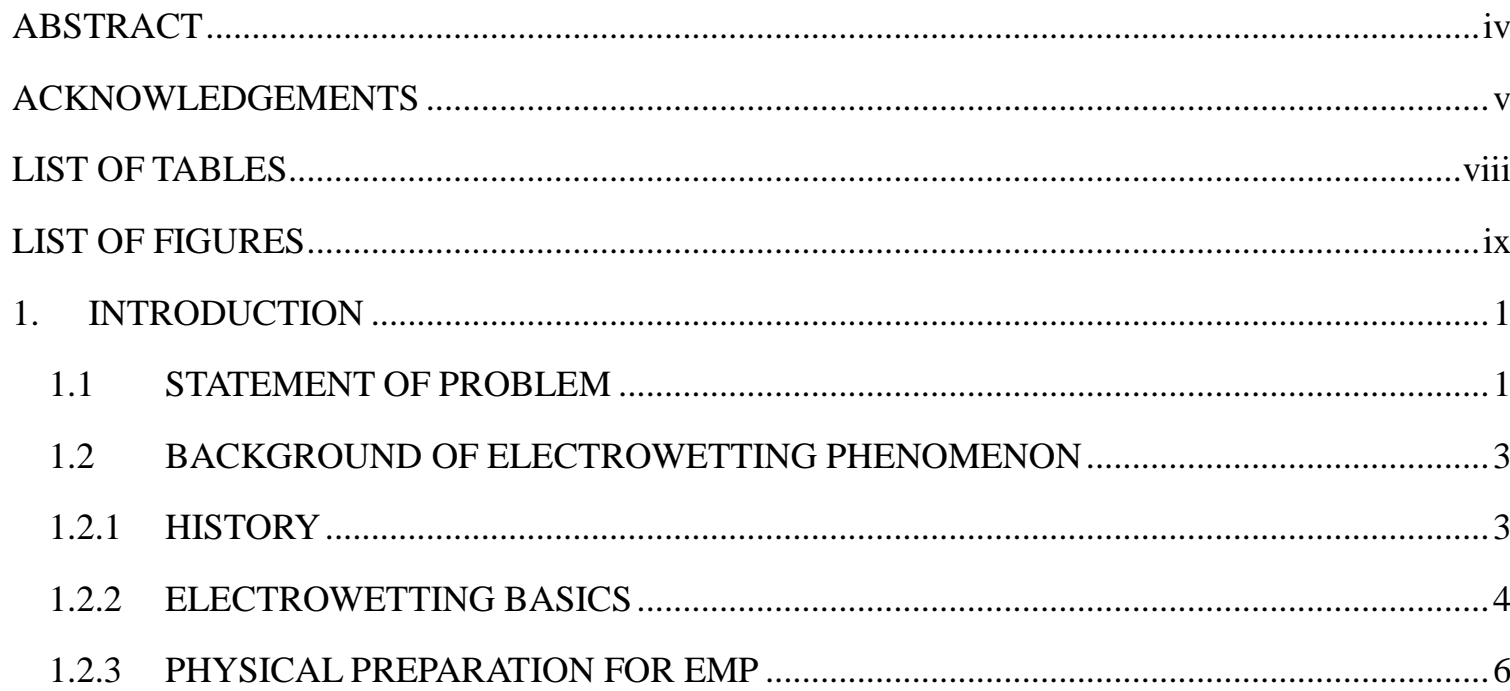

2. ELECTROWETTING MICROPRISM ARRAYS MODELING AND BEAM PROPAGATION ALGORITHM.

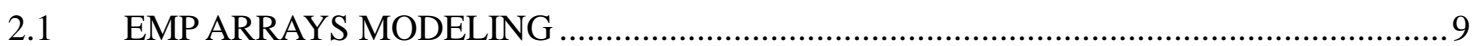

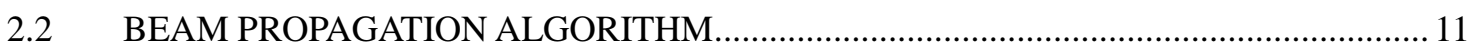

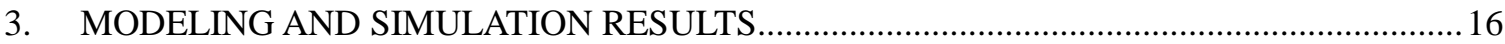

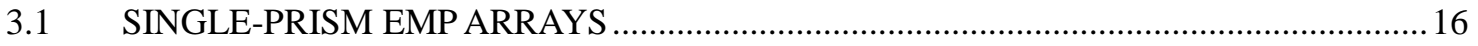

3.1.1 1D SINGLE-PRISM EMP ARRAYS AND INTRODUCTION OF TILT ............................ 16

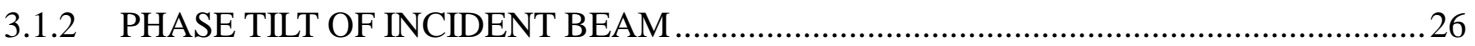

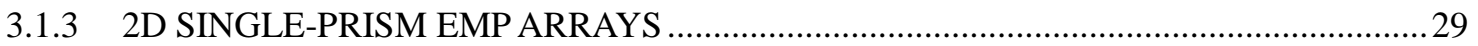

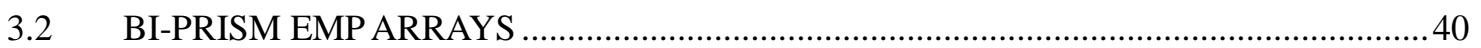

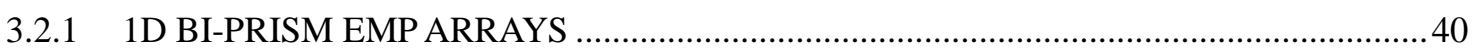

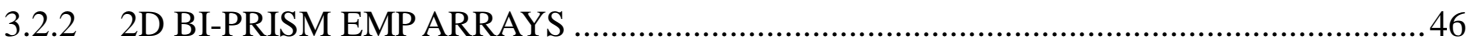

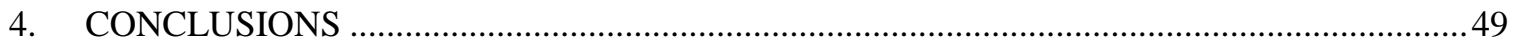




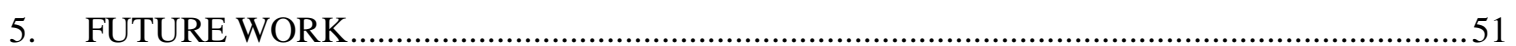

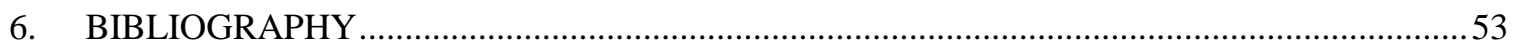




\section{LIST OF TABLES}

Table 1: Comparison of single-prism and bi-prism structures. 


\section{LIST OF FIGURES}

Figure 1-1: Comparison of a droplet on insulator coated electrode without and with applied actuation voltage. 5

Figure 1-2: Theoretical plot of voltage versus contact angle. ….................................................

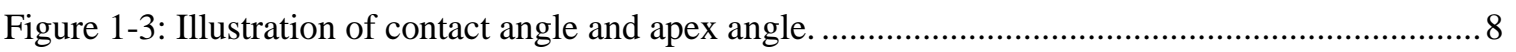

Figure 2-1: Two-dimensional geometry of single-prism EMP arrays ............................................... 10

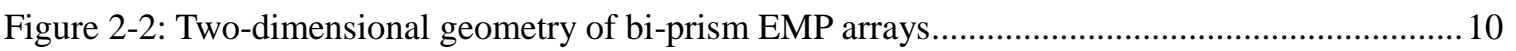

Figure 2-3: Three-dimensional view of $2 \times 2$ single-prism EMP arrays. ............................................ 11

Figure 2-4: (a) Our simulation of the far-field pattern from a liquid crystal OPA and (b) the

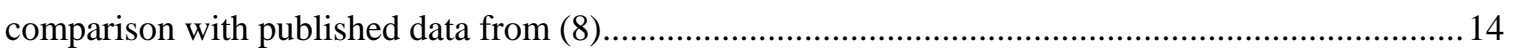

Figure 3-1: Gaussian distributed incident beam........................................................................... 17

Figure 3-2: Intensity distribution in one pixel of single-prism EMP with beam size of 40um ............. 17

Figure 3-3: Intensity distribution in 10 pixels of single pixel EMP with beam size of 200um............. 18

Figure 3-4: Far-field and near-field intensity patterns for 1D single-prism arrays at different incident angles.

Figure 3-5: 3D mesh plot with contour, $x$-axis is the far-field angle and $y$-axis is the interface angle of the 2 liquids and z-axis is the logarithm of normalized intensity at corresponding far-field angle.......22 Figure 3-6: Far field angle and interface angle dependence for single-prism EMP arrays ..................23

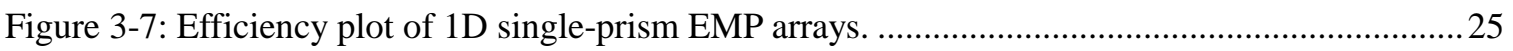
Figure 3-8: Comparison of predicted maxima based on Eq. (16) and simulation results based on beam

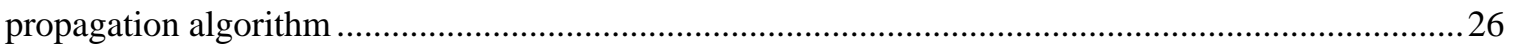

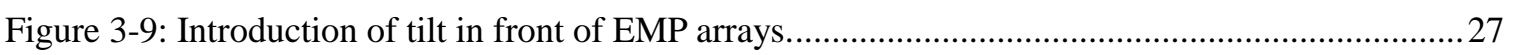

Figure 3-10: Efficiency plot from local maxima with tilt increment of 0.1 degree...............................28

Figure 3-11: Efficiency plot from local maxima with tilt increment of 0.05 degree.............................29

Figure 3-12: Optimized efficiency plot with tilt from higher peaks for 1D single-prism EMP arrays.. 29 Figure 3-13: Near-field and far-field intensity for 10x10 2D single-prism EMP Arrays for various

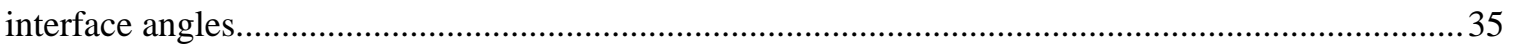

Figure 3-14: Far-field intensity pattern when I >0.368 for 10x10 2D single-prism EMP arrays. ..........36

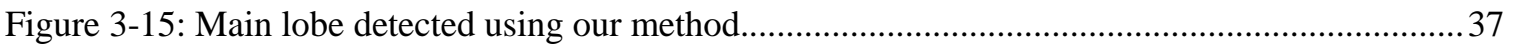

Figure 3-16: Calculated efficiency and interface angle dependence for 2D single-prism EMP arrays. 38

Figure 3-17: Far-field efficiency and interface angle dependence for 2D single-prism EMP arrays.... 39

Figure 3-18: 2D EMP arrays efficiency with tilt for 2D single-prism EMP arrays. .39 
Figure 3-19: Intensity distribution inside a bi-prism EMP pixel at interface angle $\boldsymbol{\phi} \boldsymbol{x}=\boldsymbol{\phi} \boldsymbol{y}=\mathbf{1 5}^{\circ} .40$ Figure 3-20: Intensity distribution inside 20 bi-prism pixels with beam waist of 200um..................... 41

Figure 3-21: Near-field intensity for 1D bi-prism EMP arrays at $\boldsymbol{\phi} \boldsymbol{x}=\boldsymbol{\phi} \boldsymbol{y}=\mathbf{1 5}^{\circ}$...........................42

Figure 3-22: Far-field intensity for 1D bi-prism EMP arrays at $\boldsymbol{\phi} \boldsymbol{x}=\boldsymbol{\phi} \boldsymbol{y}=\mathbf{1 5}^{\circ}$............................. 42

Figure 3-23: 3D mesh plot with contour of far-field intensity with respect to far-field and interface angle for 1D bi-prism EMP arrays in logarithm scale.

Figure 3-24: Far field angle and interface angle dependence for bi-prism EMP arrays in normalized scale. 44

Figure 3-25: Efficiency plot with respect to far-field angle for 1D bi-prism EMP arrays...................... 45

Figure 3-26: Efficiency plot of 1D bi-prism EMP arrays with phase tilt. .......................................... 45

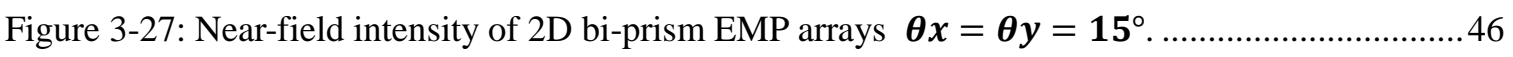

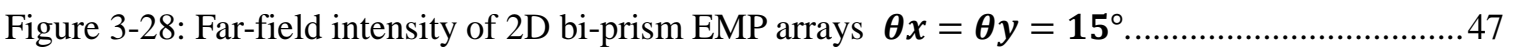

Figure 3-29: Efficiency plot with respect to far-field angles for 10x10 2D bi-prism EMP arrays........ 48

Figure 3-30: Efficiency plot of 2D bi-prism EMP arrays with phase tilt. .........................................48 


\section{INTRODUCTION}

\subsection{STATEMENT OF PROBLEM}

Beam steering techniques play an important role in many optical remote sensing applications. In traditional mechanical steering an optical system is attached to a gimbaled mount. This system requires costly and heavy mounts and driving motors to control the pointing direction along two axes. Steering is required for large telescopic optics and for remote sensing systems that need to survey a wide field of view. However, it is complicated and can be prone to mechanical failure. An elegant approach to beam steering is to use a non-mechanical system where the beam is steered by electrically controlling the wavefront of the optical beam. Development of reliable and wide angle non-mechanical beam steering devices is a long standing problem in the remote sensing field.

When a liquid electrolyte comes in contact with a solid, a contact angle is formed between the liquid meniscus and the solid surface. When the solid surface is a non-conducting layer an applied voltage can reduce the contact angle; this is the electrowetting phenomenon. In other words, we can manipulate the contact angle continuously by controlling the electrical voltage applied. The voltage-contact angle relationship has been thoroughly studied and it is proportional to the square of the voltage. 
Electrowetting optics research has mostly been devoted to the area of tunable focal length lens systems and display technologies. Electrowetting has shown potential for applications to non-mechanical beam steering, since the contact angle modulation in electrowetting effect can be used to reconfigure the liquid surface shape and therefore deflect the incident light beams. We apply this effect to beam steering technique with Electrowetting Microprism (EMP) structure.

In this chapter, I will start by giving a brief introduction to the electrowetting phenomenon. Then the single-prism and bi-prism EMP structures are described in detail. In Chapter 2 I will present the extended beam propagation algorithm we proposed using the Fast Fourier Transform (FFT) technique. Our extended beam propagation method is not restricted to paraxial beams since we employ a spatial frequency disersion relation without neglecting any higher order terms. The results are compared with the case with only the first nonzero order transverse wave evectir correction to the propagation constant. Maxwell's equations are solved in both single-prism and bi-prism geometries with input beams whose width can be one or many pixels wide. The single-prism and bi-prism EMP arrays are evaluated in both 1D and 2D structures with beam steering efficiency calculations. For comparison our numerical computation is applied to a liquid crystal array that has been reported in the literature using a finite-difference time-domain algorithm. The liquid crystal computation results are in good agreement with the results in the literature, thus giving us confidence when the results are extended to electrowetting computations. 
For the EMP arrays the diffraction angles are discrete and the angles between the diffraction peaks can be covered by applying a phase tilt to the input beam.

\subsection{BACKGROUND OF ELECTROWETTING PHENOMENON}

\subsubsection{HISTORY}

Electrowetting, i.e. the phenomenon that modifies the spreading of liquid on a substrate by applying electrostatic charge on the liquid, is a microfludic phenomenon that was first studied by Lippmann in 19th century when he discovered that externally applied electrostatic charge can modify the capillary forces on the interface by finding the capillary phenomenon at mercury-electrolyte interface [1]. At that time, the term "electrocapillarity" was coined. In 1930s, Froumkine found that the metallic surface with electric potential also "wets" electrolyte against air [2]. The term electrowetting was officially adopted in the 1980s to describe a property needed for a new display technology design. People found that electrowetting is caused by forces tangential to the interface in terms of surface tension variation, which is essentially due to the effects of the applied electric field, while the electrocapillarity effect is due to the forces normal to the interface. However, people hadn't spent a lot of time enhancing or utilizing this phenomenon until recently when the great potential of electrowetting in optics and other applications was discovered. Berge proposed a structure called electrowetting on insulator coated electrodes (EICE) in 1993, whose robustness, reversibility and low-cost made itself a promising candidate for many applications [3]. The main advantage is that the voltage required for driving contact angle 
on EICE is much lower than the breakdown voltage for insulator, which allows much larger contact angle variation, up to $80^{\circ}$, compared to a few tenths of degrees contact angle control [4] [5]. Our model is essentially based on EICE structure.

\subsubsection{ELECTROWETTING BASICS}

Starting from metal-electrolyte, Lippmann has characterized the relationship as:

$$
\frac{d \gamma_{S L}}{d V}=-\sigma
$$

where $\gamma_{S L}$ is the solid-liquid interfacial tension, $\mathrm{V}$ is the potential difference across the interface of metal and electrolyte and $\sigma$ is the surface charge density. Berge further modified the equation for partially wetting case, i.e. the EICE, by combining the derivative of Eq. (1) with Young Equation, yielding:

$$
\frac{d^{2} \cos \theta}{d V^{2}}=\frac{c}{\gamma}
$$

where $\theta$ is the contact angle of the liquid on insulator, $\gamma$ again is the liquid surface tension and $\mathrm{c}$ is the measurable solid-liquid capacitance [6]. Starting from Young-Lippmann equation, Berge has concluded the relationship between the contact angle $\theta(V)$ in electrowetting and the applied voltage $\mathrm{V}$ as follows,

$$
\cos \theta=\cos \theta_{0}+\frac{c}{2 \gamma} V^{2}
$$

where $\mathrm{V}$ is the applied DC or AC (RMS) voltage, $\theta_{0}$ is Young's contact angle at no voltage which is determined by the surface hydrophobicity, $\mathrm{c}$ is the capacitance per unit

area of the Parylene/fluoropolymer $\left(\mathrm{F} / \mathrm{m}^{2}\right)$, and $\gamma$ is the interfacial surface tension between 
the aqueous and oil phases $(\mathrm{N} / \mathrm{m})$. For EICE, the capacitance $\mathrm{c}$ is on the order of $100 \mathrm{pF} / \mathrm{m}^{2}$ and $\gamma$ for pure water is $73 \mathrm{mN} / \mathrm{m}$.

The basic process of EICE is demonstrated in Figure 1-1 below.
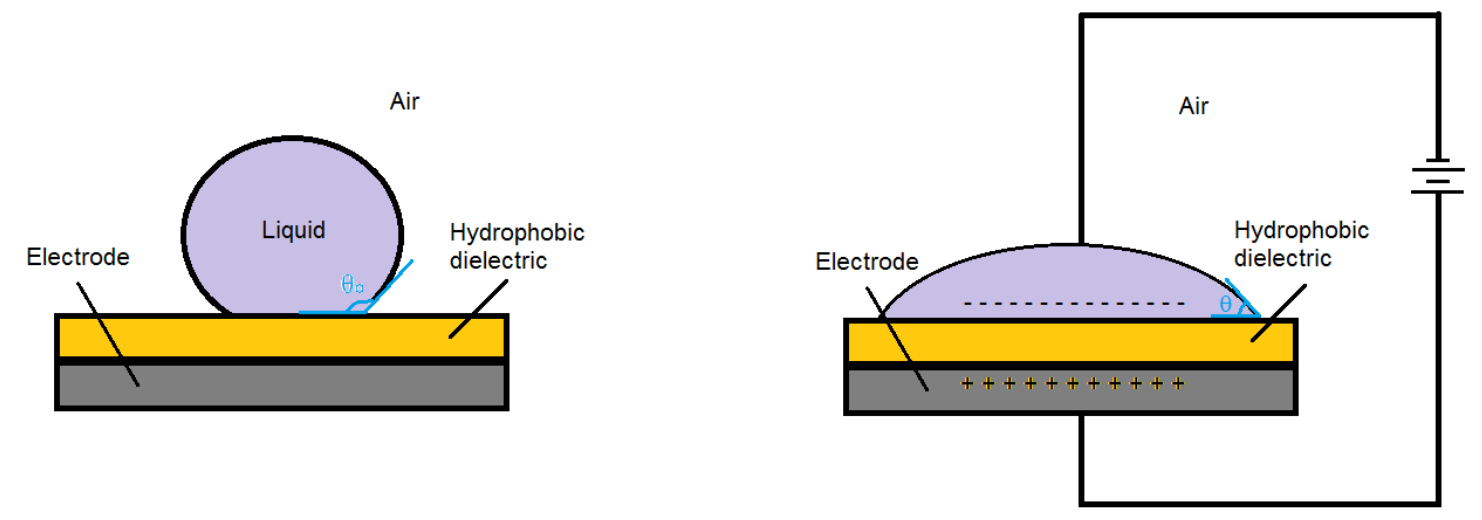

Figure 1-1: Comparison of a droplet on insulator coated electrode without and with applied actuation voltage.

In Figure 1-1, a droplet of aqueous solution in ambient air is deposited on a hydrophobic dielectric with a base electrode underneath. Since the amount of water is very small, the surface tension is dominant over gravity or other effects in terms of surface shape. When no electric field is applied, the hydrophobic surface of the dielectric gives rise to an initial contact angle $\theta_{0}>90^{\circ}$. However, once a voltage is applied across the insulator, the induced charge which makes the insulator a parallel capacitor with liquid and electrode serving as two parallel conducting plates and so-called solid-liquid capacitance c, is going to break the equilibrium of the chemical and electrical components of Gibbs free energy, which further changes the surface tension.

Electrowetting is defined as a rapid manipulation of the contact angle between the solid hydrophobic surface and electrolyte by applying an electric field across the surface and 
liquid so as to reduce the surface tension, making the hydrophobic surface wettable, in other word, more hydrophilic. It is also predicted by theory that the flow rate in electrowetting could reach $10 \mathrm{~cm} / \mathrm{sec}$ for $1 \mathrm{~V}$ actuation voltage, which is very fast given the fact that the device size is typically on the order of millimeters or microns. This property enables electrowetting as a promising technique for optical switches and optical display device.

\subsubsection{PHYSICAL PREPARATION FOR EMP}

The electrowetting device for beam steering application was proposed by Dr. Jason Heinkenfeld's group. This is the group that we are collaborating with. Water with KCL is chosen to be the conducting liquid for our electrowetting applications [7]. An immiscible mixture of oil and the salt water was used in our designs. Oil is selected for its higher refractive index, water as solvent for its stable property with light and KCL is to provide sufficient metal ions to enable the wetting phenomenon [7]. Heikenfeld's group found that since matter in the liquid form is more dispersive than its solid form, a two-liquid (oil/water) system is preferred in terms of spatial dispersion related applications as long as the density match is achieved [7]. In our modeling, a fluoropolymer is dip coated with a thickness of $\sim 1 \mu \mathrm{m}$ and $\varepsilon_{\mathrm{r}}$ of 1.9. The ratio of oil, water and KCL is chosen that the surface tension $\gamma$ is very close to that of pure water, which is 73 dynes/cm. The Young's contact angle $\theta_{0}$ is found to be $115^{\circ}$ [7]. With all the parameters given, we can simplify Eq. (3), yielding:

$$
\cos \theta=-0.4226+1.1522 \times 10^{-4} V^{2}
$$


The relationship between contact angle $\theta$ and applied voltage $\mathrm{V}$ can be plotted in Figure 1-2 below:

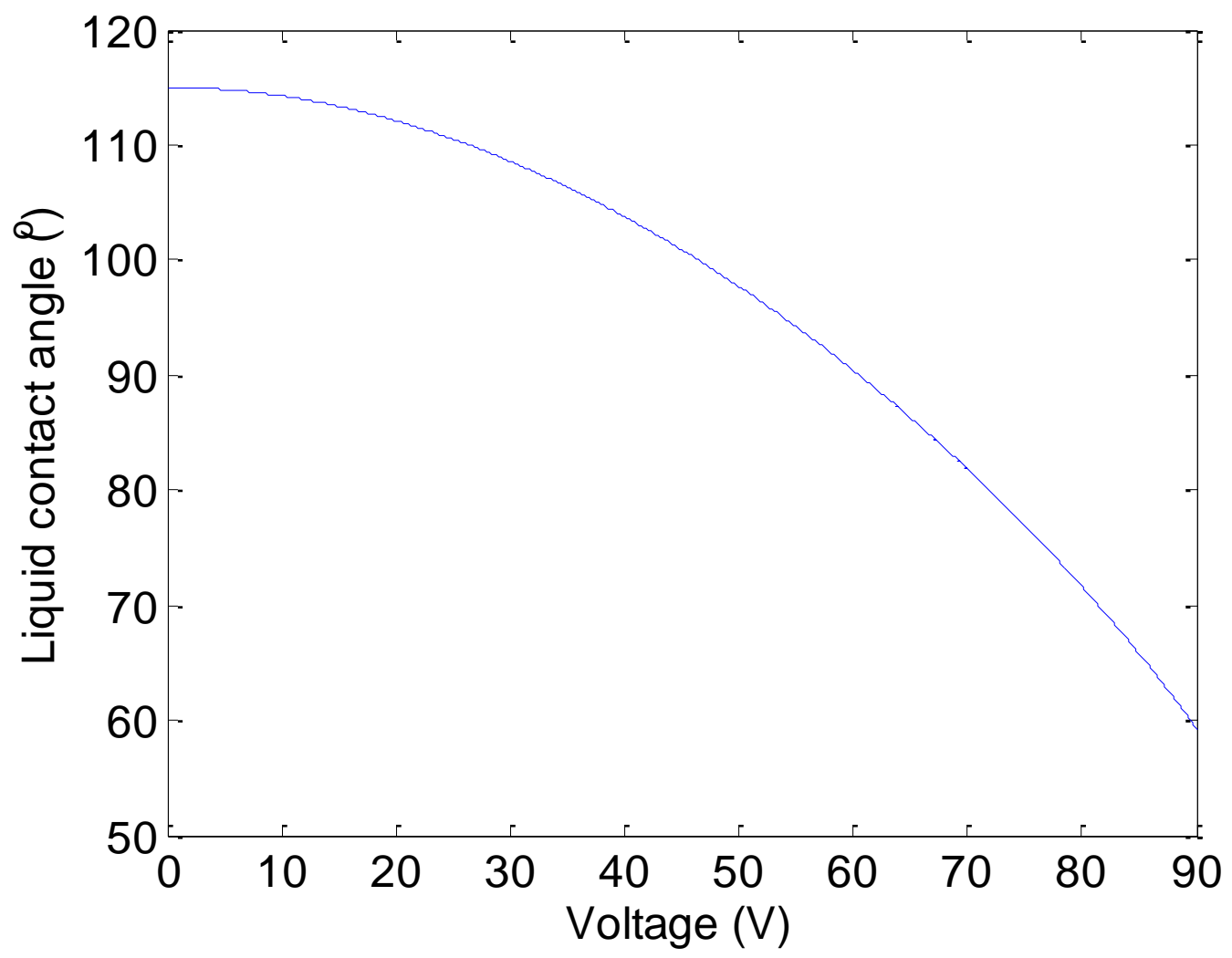

Figure 1-2: Theoretical plot of voltage versus contact angle.

Due to the saturation, the contact angle can only go down to $70^{\circ}$, which actually confines the usable contact angles to be from $70^{\circ}$ to $110^{\circ}$ due to the fact that two contact angles from two parallel sidewalls have to add to $180^{\circ}$ in order to maintain a flat surface. This implies that in our design, an independent voltage source is required for each sidewall because different voltages will be needed for two sidewalls at the same time. If we compare this to a prism, the apex angle is equal to $\left|\theta-90^{\circ}\right|$. This is illustrated in Figure 1-3. 


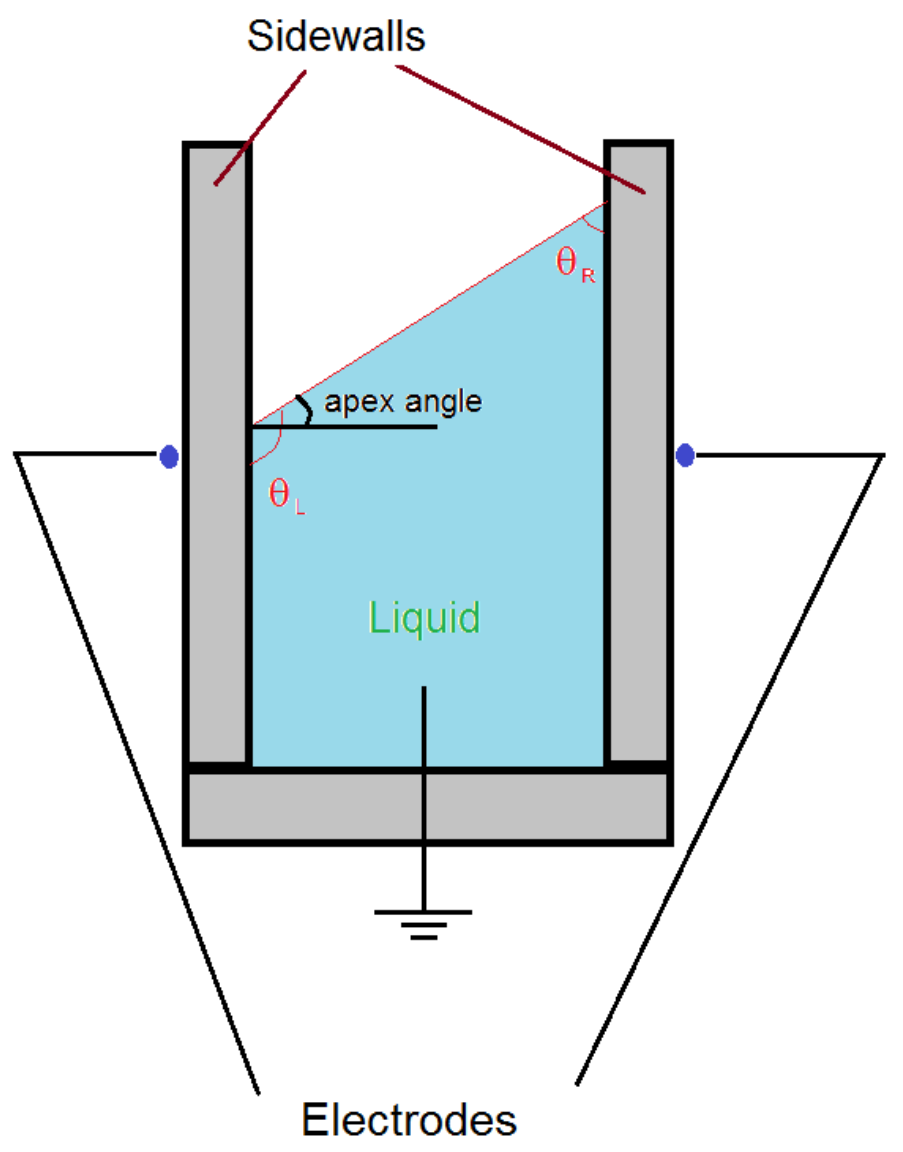

Figure 1-3: Illustration of contact angle and apex angle.

The scaled-down Electrowetting Microprism, on the order of microns, is designed to further increase the steering speed and surface flatness. This model will be extensively studied in the following chapters. Also research on other higher refractive index liquid and higher hydrophobicity dielectric is currently being done to further improve the performance. 


\section{ELECTROWETTING MICROPRISM ARRAYS MODELING AND BEAM PROPAGATION ALGORITHM}

\subsection{EMP ARRAYS MODELING}

Figure 2-1 and Figure 2-2 illustrates the 2D geometry of single-prism and bi-prism EMP structure, respectively. The parameters for both of the structures based on Dr. Jason Heikenfeld's group are specified in the following table.

Table 1: Comparison of single-prism and bi-prism structures.

\begin{tabular}{|c|c|c|}
\hline Parameters & $\begin{array}{c}\text { Single-prism EMP Arrays } \\
\text { (1D and 2D) }\end{array}$ & $\begin{array}{c}\text { Bi-prism EMP Arrays (1D } \\
\text { and 2D) }\end{array}$ \\
\hline Side wall & $47 \mu \mathrm{m}$ & $80 \mu \mathrm{m}$ \\
\hline Bottom layer of glass & $60 \mu \mathrm{m}$ & $90 \mu \mathrm{m}$ \\
\hline Top layer of glass & $70 \mu \mathrm{m}$ & $100 \mu \mathrm{m}$ \\
\hline Wall width & $2 \mu \mathrm{m}$ & $2 \mu \mathrm{m}$ \\
\hline Pixel width & $52 \mu \mathrm{m}$ & $52 \mu \mathrm{m}$ \\
\hline Refractive index of water & 1.3 & 1.3 \\
\hline $\mathrm{n}_{\text {glass }}=\mathrm{n}_{\text {oil }}$ & 1.6 & 1.6 \\
\hline
\end{tabular}




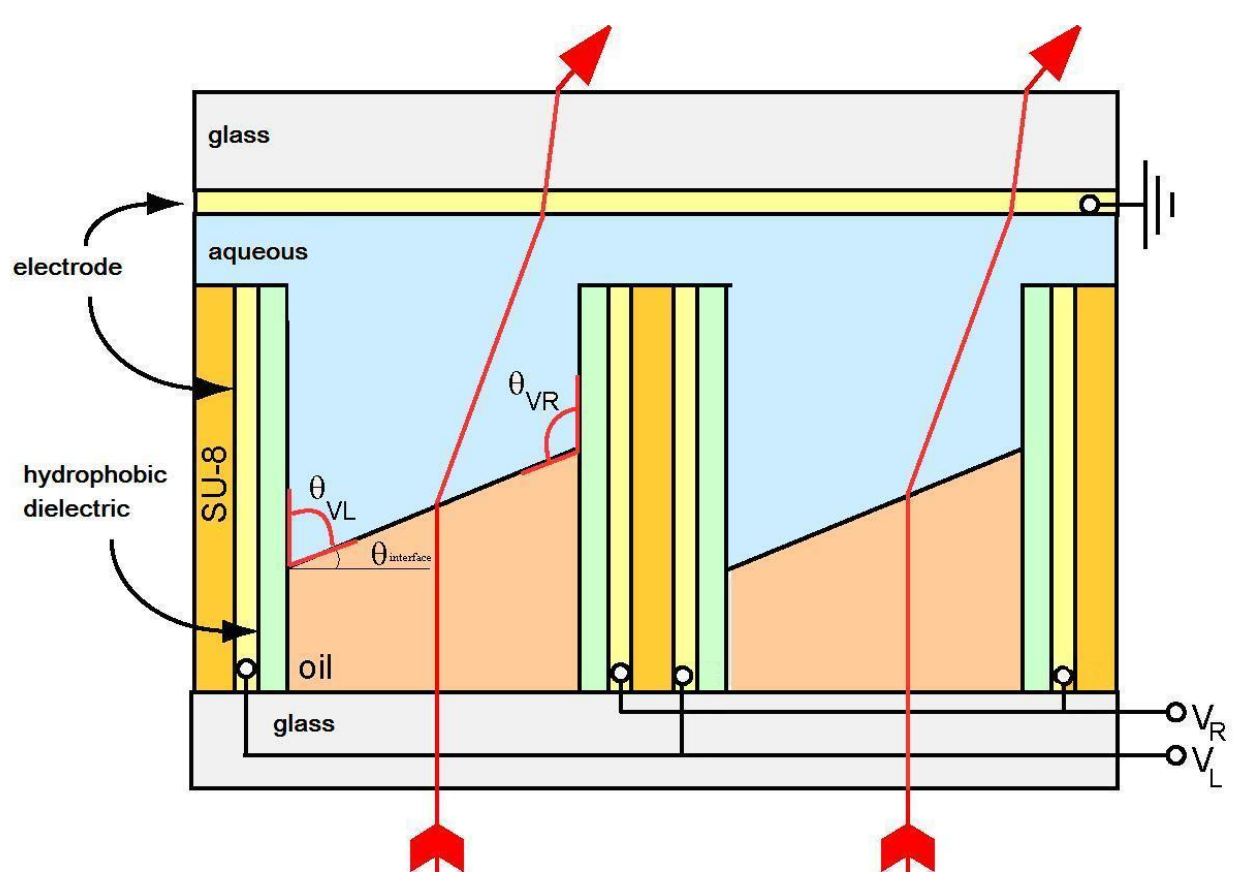

Figure 2-1: Two-dimensional geometry of single-prism EMP arrays

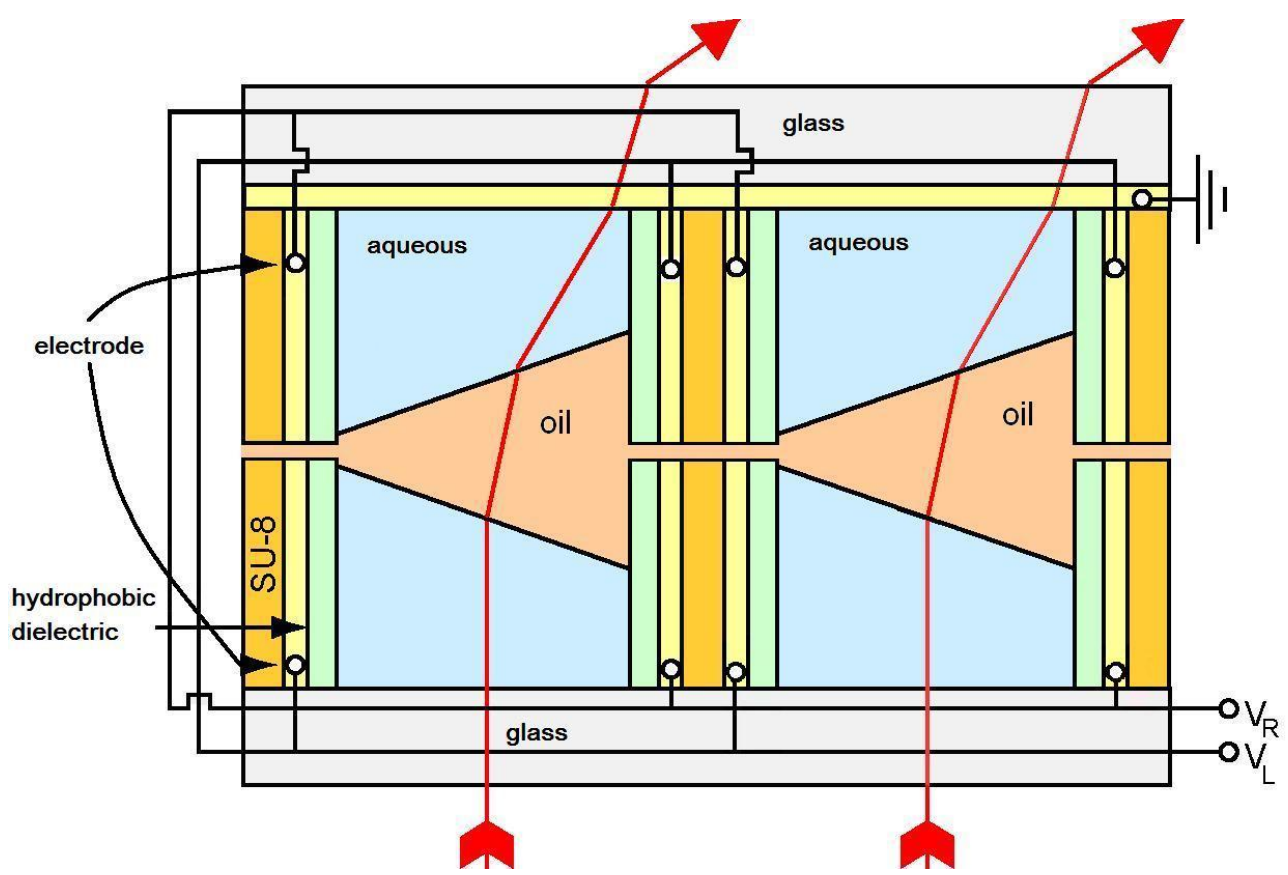

Figure 2-2: Two-dimensional geometry of bi-prism EMP arrays 


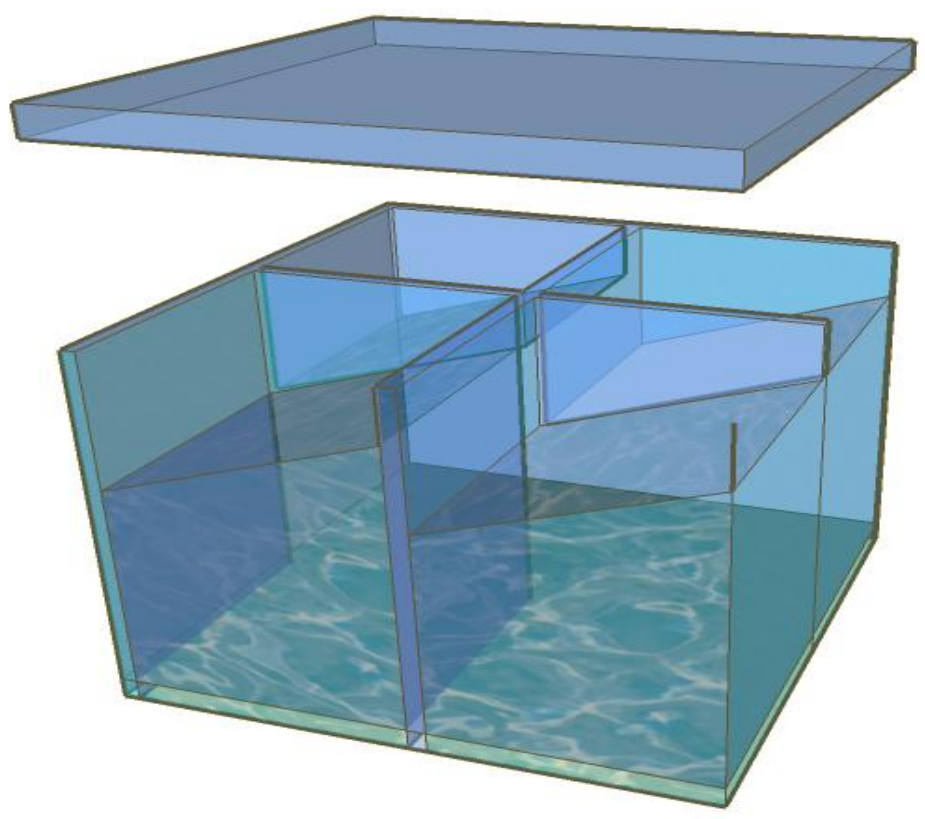

Figure 2-3: Three-dimensional view of $2 \times 2$ single-prism EMP arrays.

Figure 2-3 demonstrates a 3D view of 2x2 EMP arrays. As indicated in previous discussion, an oil/water configuration is used to create the linear phase change and the surface of the side walls is coated with a fluoropolymer (a hydrophobic material) to ensure a large contact angle for the water/side wall interface.

\subsection{BEAM PROPAGATION ALGORITHM}

From a physical point of view, the refractive index difference as a function of position at the oil/aqueous interface deflects the incident beam, which adds a linear phase change to the wavefront. When a linear phase tilt is applied along the $\mathrm{x}$-axis, the wave vector has a transverse $\mathrm{x}$-component, thus deflecting the beam. The ratio of the $\mathrm{x}$-component of the wave vector to the propagating direction component $(\mathrm{z})$ gives rise to the deflection angle. 
In our modeling, we start from the Helmholtz electromagnetic wave equation with refractive index as a function of location $\mathrm{x}$ and $\mathrm{z}$,

$$
\frac{\partial^{2} E}{\partial z^{2}}+\frac{\partial^{2} E}{\partial x^{2}}+n^{2}(x, z) k_{o}^{2} E=0
$$

where $\mathrm{n}(\mathrm{x}, \mathrm{z})$ is the refractive index as a function of both $\mathrm{x}$ and $\mathrm{z}$ and $\mathrm{n}_{\mathrm{b}}$ is the background refractive index where we choose to start propagation. $\mathrm{k}$ is the wave number of the propagating beam in air and $\mathrm{k}_{\mathrm{o}}$ is the wave number in glass/oil.

For a constant refractive index, i.e. $n(x, z)=n$, we can Fourier transform the equation into spatial frequency domain with respect to $\mathrm{x}$, yielding,

$$
\frac{\partial^{2} \tilde{E}}{\partial z^{2}}+\left(n^{2} k_{o}^{2}-k_{x}^{2}\right) \tilde{E}=0
$$

The solutions can be found as,

$$
\tilde{E}=E_{+} e^{i \sqrt{n^{2} k_{o}^{2}-k_{x}^{2}} z}+E_{-} e^{-i \sqrt{n^{2} k_{o}^{2}-k_{x}^{2}} z}
$$

The E. which represents the reflected electric field, is commonly ignored since it is only in the order of

$$
\left(\frac{\Delta n}{n}\right)^{2} \approx 3.5 \% \text {. }
$$

When $\mathrm{n}$ is not a constant, normally we use paraxial approximation, $E=\hat{E} e^{i k z}$, where $k=\bar{n} k_{o}$, yielding,

$$
\frac{\partial \hat{E}}{\partial z}=\frac{i}{2 K} \frac{\partial^{2} \hat{E}}{\partial x^{2}}+\frac{i}{2 k} k_{0}^{2}\left(n^{2}(x, z)-n_{b}^{2}\right) \hat{E}
$$

Starting from the Eq. (9), we separate the right side into two steps in order to numerically 
solve the equation.

$$
\frac{\partial \hat{E}}{\partial z}=\frac{i}{2 k} \frac{\partial^{2} \hat{E}}{\partial x^{2}}
$$

and

$$
\frac{\partial \hat{E}}{\partial z}=\frac{i}{2 k} k_{0}^{2}\left(n^{2}(x, z)-n_{b}^{2}\right) \hat{E}
$$

It is a common numerical technique called the split-step operator method. Using this method we can calculate the electric field using the exponential form with respect to variable z. Assuming at each height $\mathrm{z}$, the electric field is a function called $A$, then Eq. (10) is Fourier transformed into spatial frequency domain with respect to $\mathrm{x}$, generating,

$$
\frac{\partial \tilde{A}}{\partial z}=-\frac{i k_{x}^{2}}{2 k} \tilde{A}
$$

where $\mathrm{k}_{\mathrm{x}}$ is the spatial frequency of $\mathrm{x}$ axis. This is solved by multiplying the electric field in spatial frequency domain by an exponential term,

$$
e^{-i \frac{k_{x}^{2}}{2 k} \cdot d z}
$$

Then we inverse Fourier transform the electric field back to space domain and which gives the answer to the first step as

$$
A=i f f t\left(f f t(A) \cdot e^{-i \frac{k_{x}^{2}}{2 k} \cdot d z}\right)
$$

Eq. (11) is numerically solved using finite difference increments in $\mathrm{z}(\mathrm{dz})$, the dielectric constant variations are constant in each slice of $\mathrm{z}$, which gives rise to the final answer in the near-field, 


$$
A=A_{0} e^{i \frac{k_{0}^{2}}{2 k}\left(n^{2}(x, z)-n_{b}^{2}\right) d z}
$$

For non-paraxial case, we replace $e^{-i \frac{k_{x}^{2}}{2 k} \cdot d z}$ in Eq. (14) with $e^{i\left(\sqrt{k^{2}-k_{x}^{2}}-k\right) \cdot d z}$, given the limit that $k_{x} \rightarrow 0$. This extension enables applications to larger diffraction angles.

In most long-distance applications such as remote sensing, we are more interested in the far-field irradiance. The far-field or Fraunhofer region is achieved by applying a Fourier transform to the near-field electric-field function. The result is used to calculate the far-field intensity and evaluate the far-field diffraction angle.

A comparison of our computational method with a finite-difference time-domain (FDTD) calculation was made. We chose a Liquid Crystal Optical Phased Array from Kent State University. Figure 2-4(a) is the result from our wave propagation approach and Figure. 2-4(b) is taken from Dr. Bos' paper using the FDTD method [8].
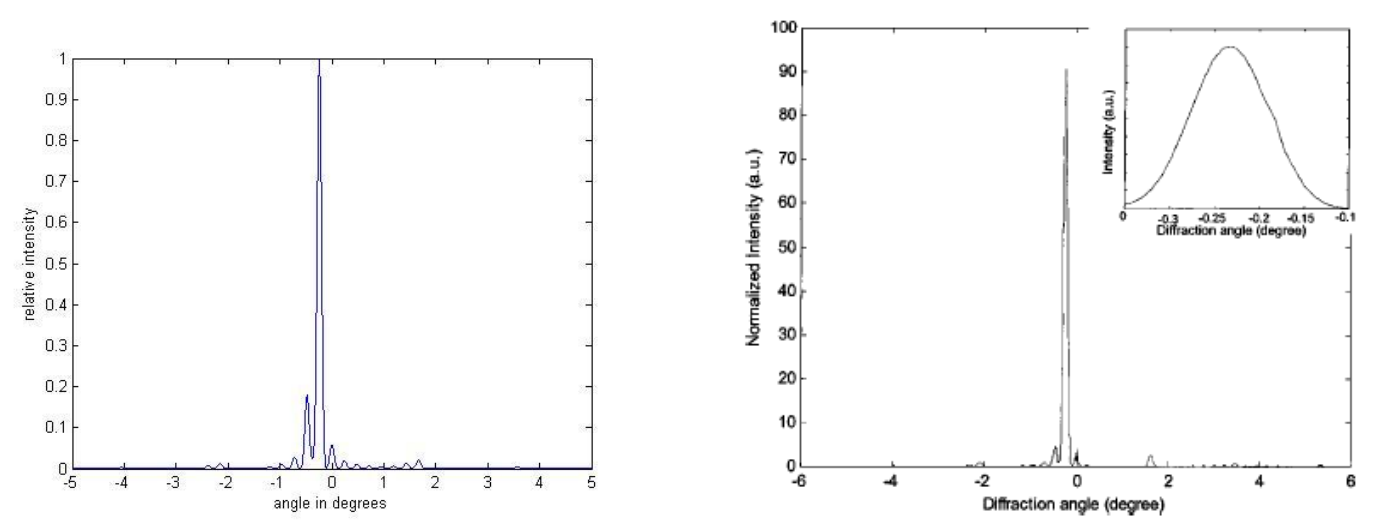

Figure 2-4: (a) Our simulation of the far-field pattern from a liquid crystal OPA and (b) the comparison with published data from [8]

The steering angle is correct and the only difference is that our approach yields a higher diffraction peak near 0.5 degrees, which results in a lower efficiency for the beam 
steering. Other than this distinction both results agree very well. This provides a greater degree of confidence that the modeling method is fully adequate for the EMP modeling, as now described in the following sections.

Electromagnetic beam propagation simulation techniques are employed to understand the operation of the EMP designs. We use an extended form of the spectral beam propagation method that can describe larger deflection angles. The method neglects reflections at each surface, but our comparison of our numerical technique with more numerically intensive methods shows that the method quantitatively captures the steered beam profile. 


\section{MODELING AND SIMULATION RESULTS}

\subsection{SINGLE-PRISM EMP ARRAYS}

\subsubsection{D SINGLE-PRISM EMP ARRAYS AND INTRODUCTION OF TILT}

Given the input Gaussian distributed beam shown in Figure 3-1, Figure 3-2 is a simulation result that demonstrates how the incident beam is deflected when propagating through a single-prism EMP cell with a 30 degree interface angle. The side wall on the left side of the figure results in a V-shaped shadow region which is due to the interference with the reflection. Figure 3-3 shown below demonstrates the intensity transition when a 200 $\mu \mathrm{m}$ wide Gaussian beam is incident into EMP arrays. The V-shaped dark bands appear on the left boundary of each pixel. The deflected light inside the sidewall on the left experiences a total internal reflection when the light enters a glass/water boundary. Simultaneously the bright spots appear also due to the sidewall where the undeflected transmitted intensity is affected by interference with beams deflected by the side walls. 


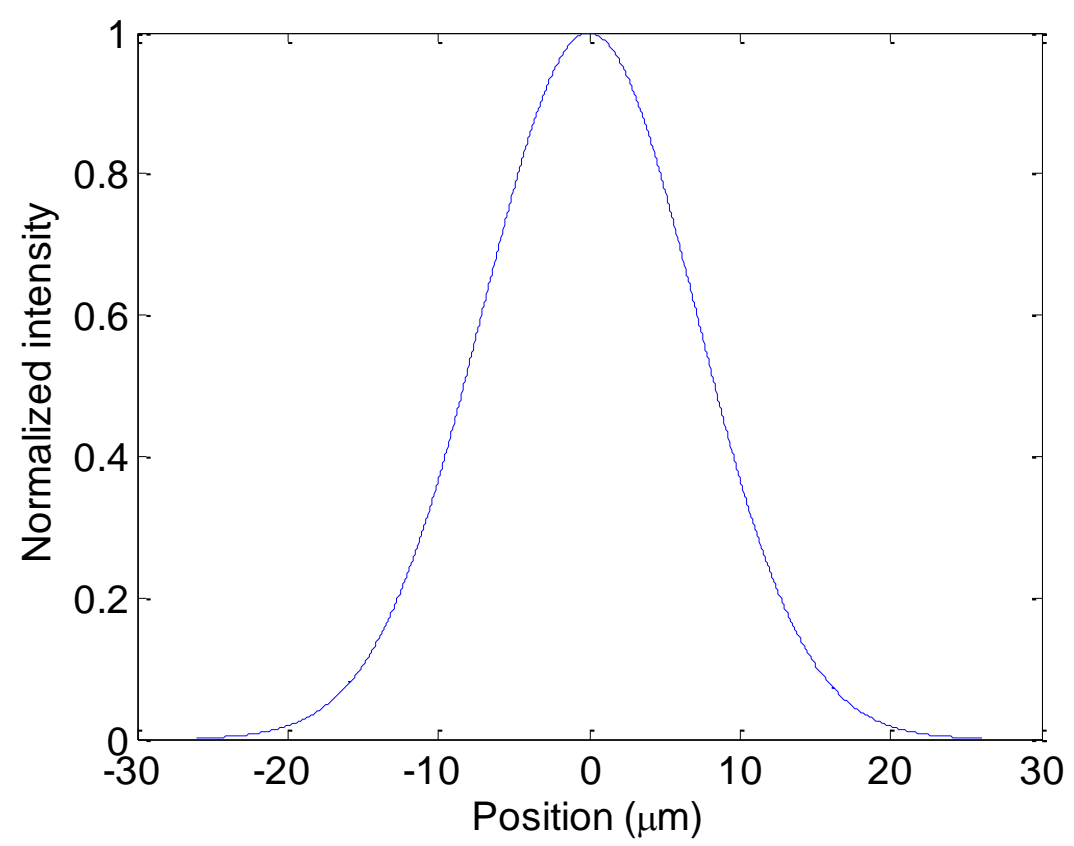

Figure 3-1: Gaussian distributed incident beam

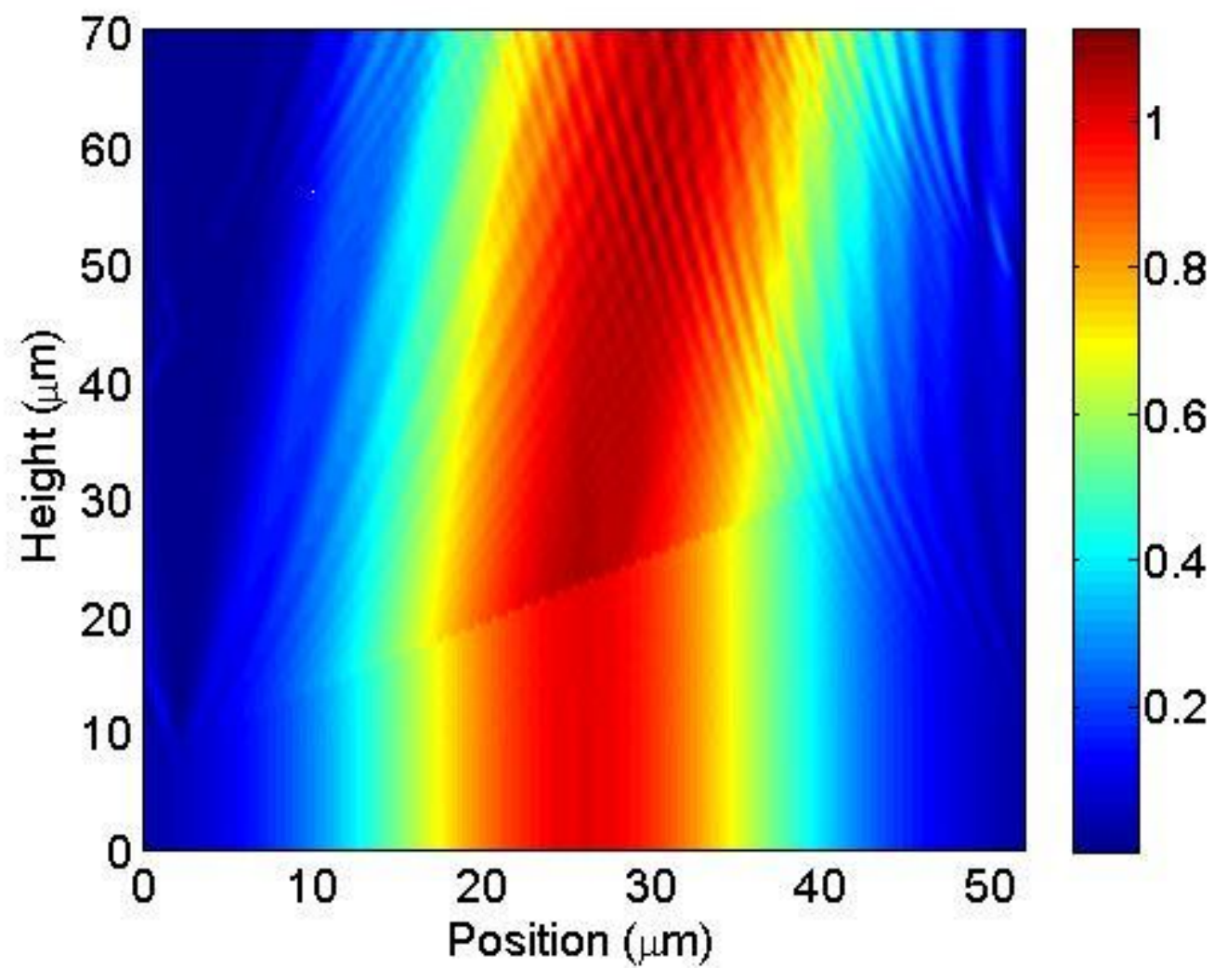

Figure 3-2: Intensity distribution in one pixel of single-prism EMP with beam size of $40 \mu \mathrm{m}$ 


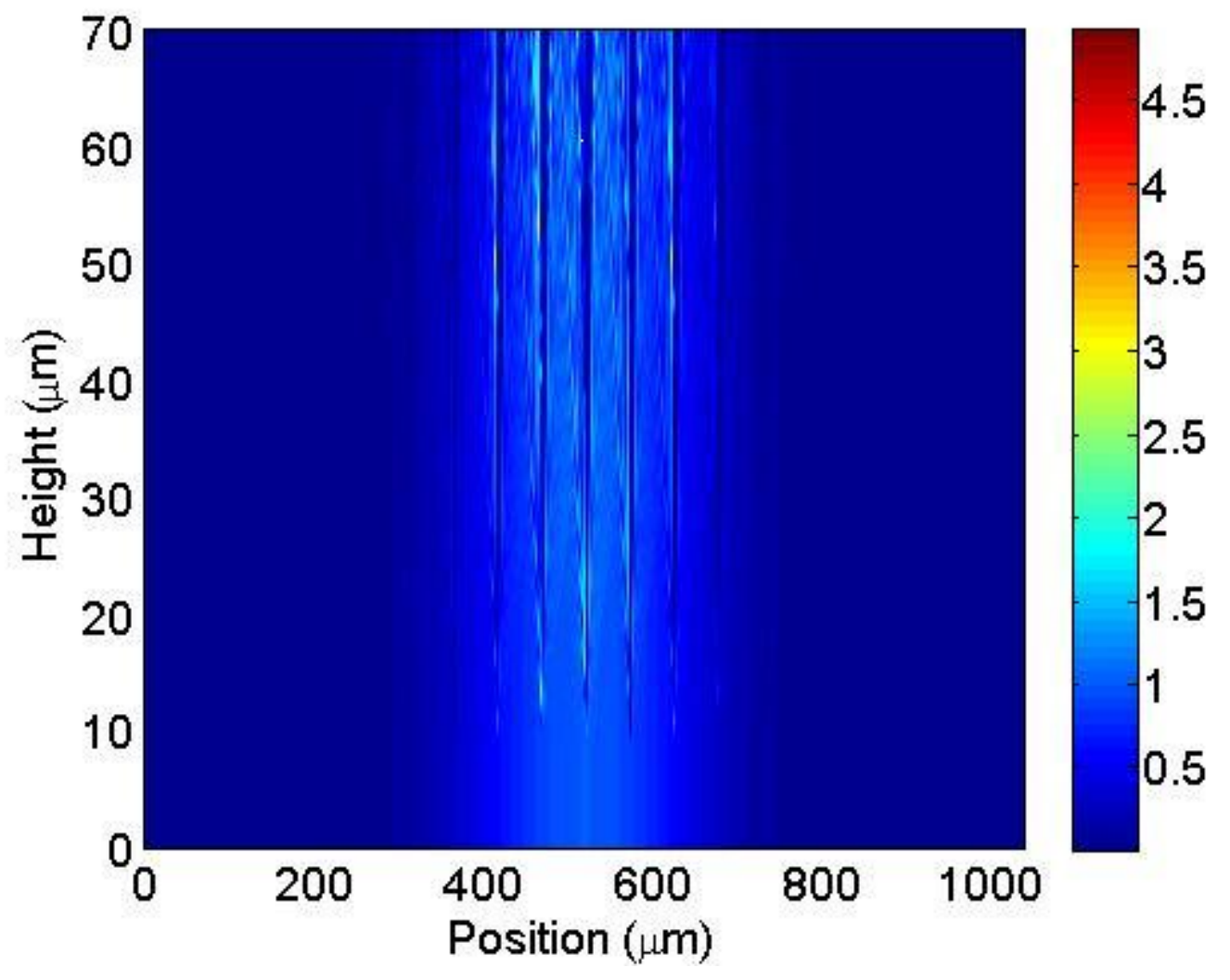

Figure 3-3: Intensity distribution in 10 pixels of single pixel EMP with beam size of $200 \mu \mathrm{m}$.

In 1D single-prism EMP Arrays case, we calculate both the near-field and far-field intensities with 20 pixels and incident Gaussian beam waist of $40 \mu \mathrm{m}$. The near-field looks rough, narrow and closely spaced interference peaks due to the sidewall interactions and pixel cross-talk in the EMP array, also the fact that there are no deflections underneath the sidewalls in each pixel. This interference also accounts for most of the energy loss to higher deflection angles in far-field. However, the far-field intensity is a lot smoother Gaussian distribution with several lobes and a reasonable amount of energy is stored in the main lobe.

Our simulations on the single-prism 1D beam steering devices are illustrated by Figure 
3-4 and are reported in this section. The paired far-field and near-field intensities are generated at discrete interface angle with a spacing of 5 degrees starting from 5 degrees. The near-field pattern has a hole in the center which becomes wider for larger interface angles and the far-field pattern has power displaced to larger diffraction angles. The hole in the near-field pattern is due to the reflection off the side wall at the peak intensity. At larger angles the exposed side wall is longer and this creates a larger hole. Arbitrarily chosen interface angles have far-field beams that are spread over several peaks for the chosen interface angles. For specific angles the energy is highly concentrated on one specific diffraction peak in the far field.

$$
\alpha=5^{\circ}
$$
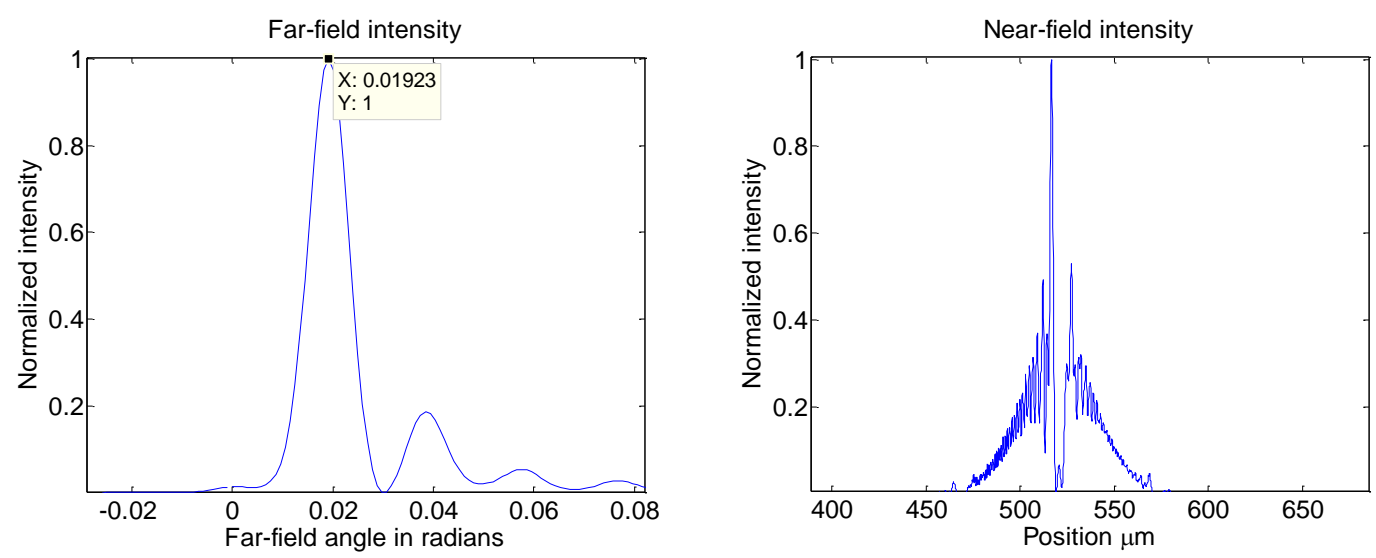

$\alpha=10^{\circ}$ 

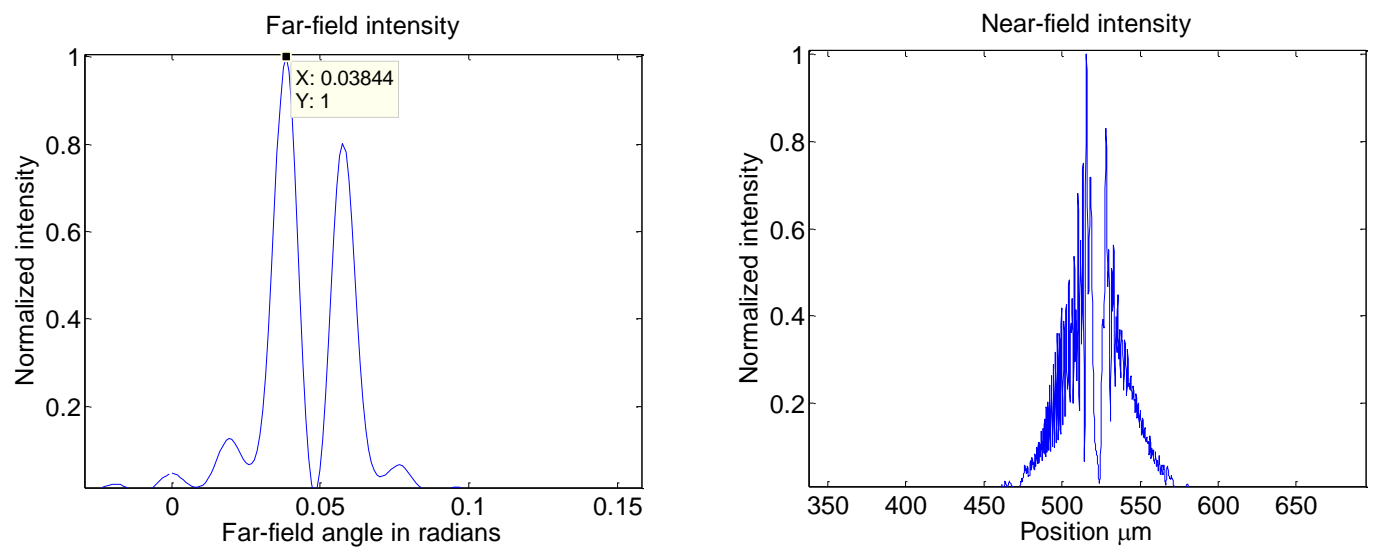

$\alpha=15^{\circ}$
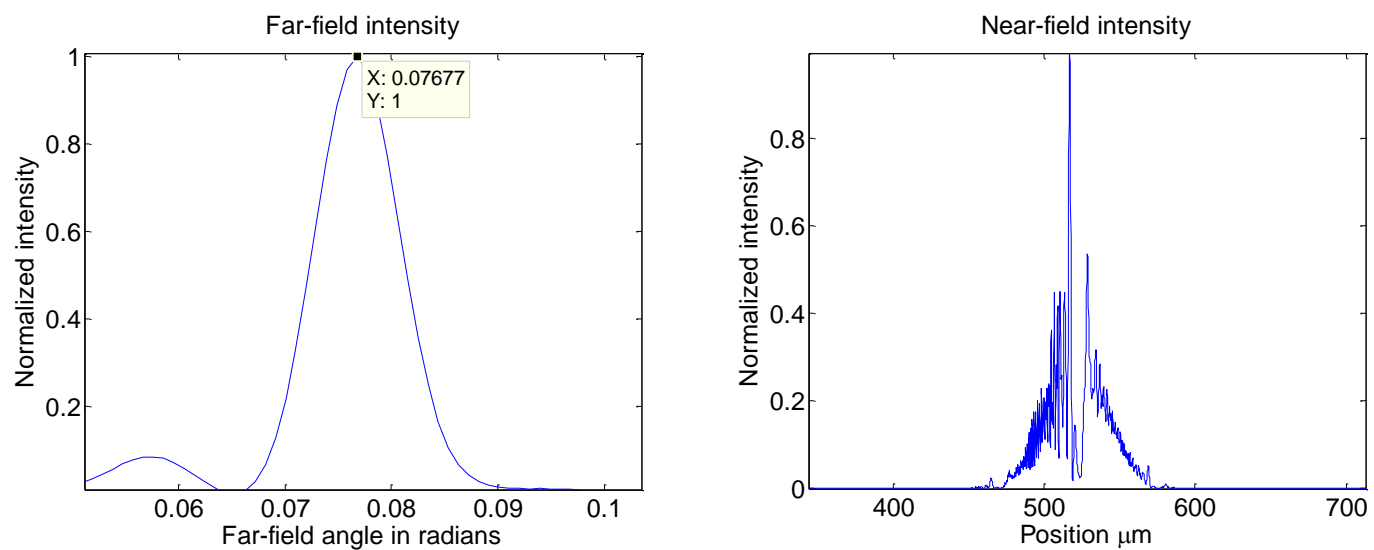

$\alpha=20^{\circ}$
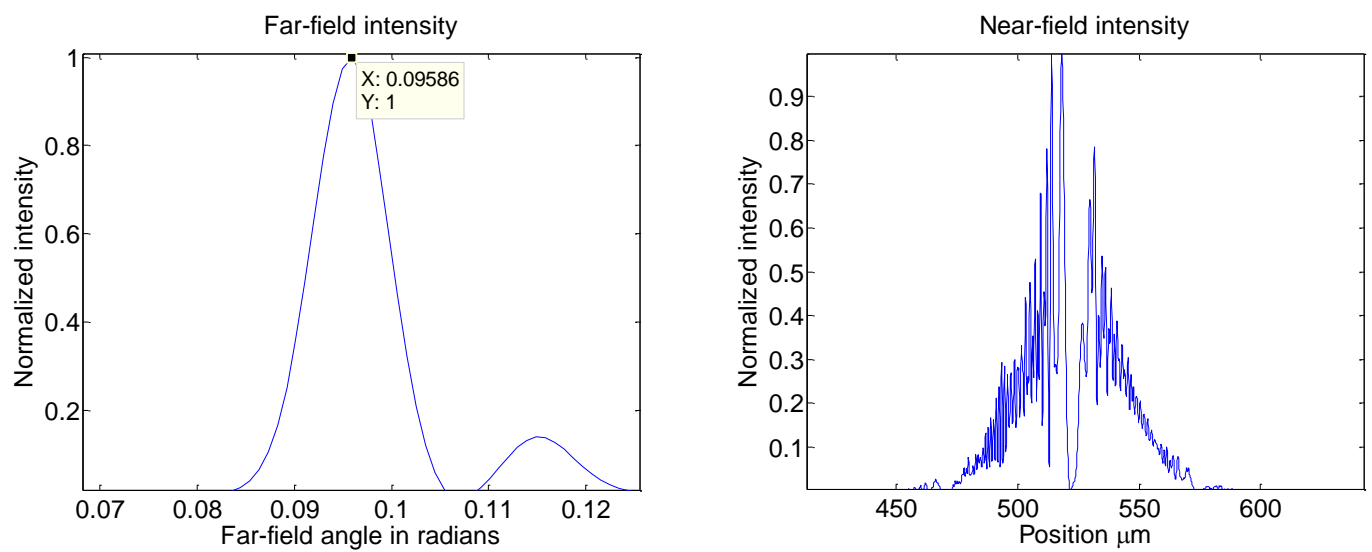

$\alpha=25^{\circ}$ 

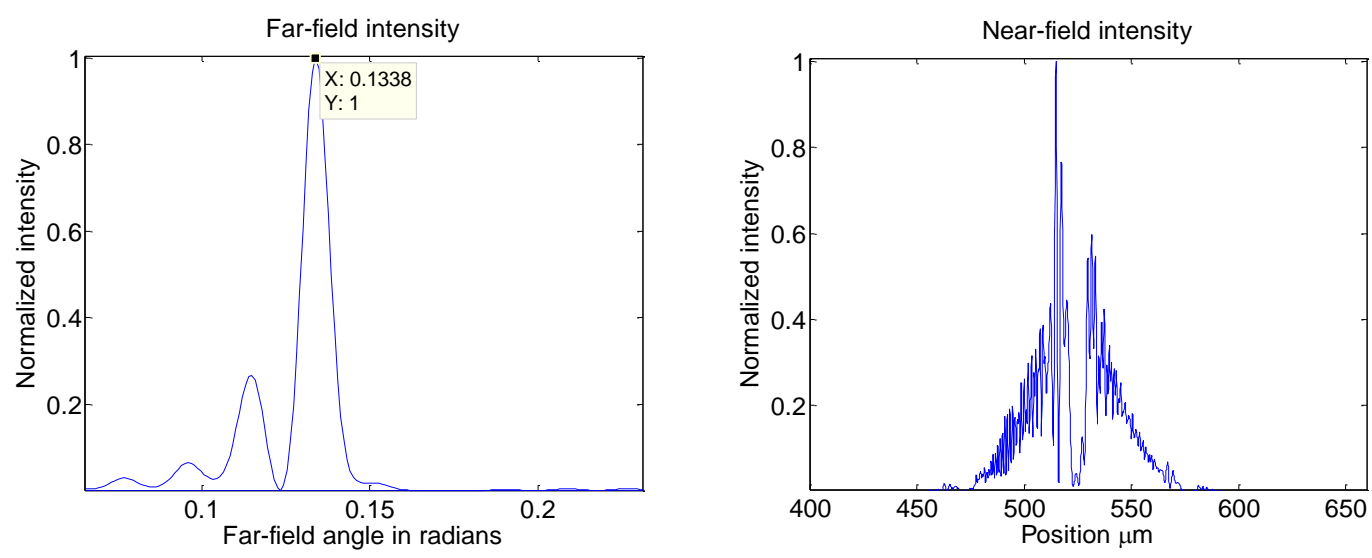

$$
\alpha=30^{\circ}
$$
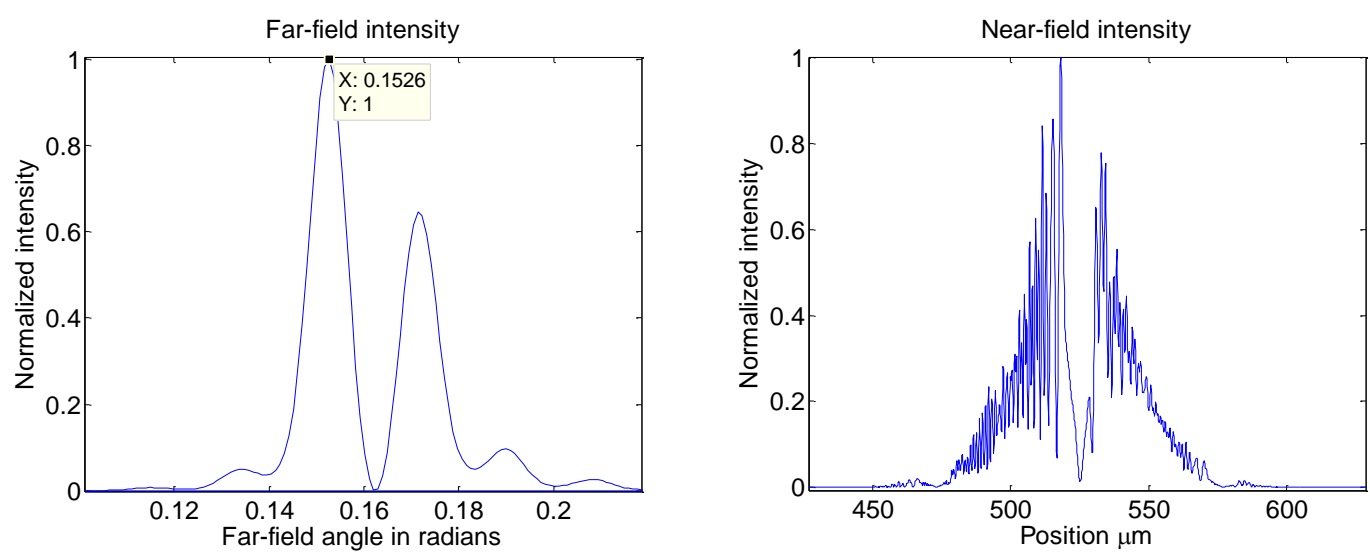

Figure 3-4: Far-field and near-field intensity patterns for 1D single-prism arrays at different incident angles.

Of all the performance parameters, efficiency is the key factor in high power beam steering applications. We use the "power-in-the-bucket" method of calculating steering efficiency. The best one can do is to steer $100 \%$ of the beam energy to the desired far-field angle. For the power in the bucket method of efficiency calculation the efficiency is defined as the intensity that falls inside the angular region defined by the first lobe divided by the total intensity over all far-field angles. We are especially interested in the relation between efficiency and interface angle. Based on our previous calculations, we find that the efficiency is fluctuating as a function of interface angles. Figure 3-5 shown below is 
the 3D plot of far-field intensity that shows the interface angle and far-field diffraction angular dependence. The diffracted intensity has a narrow maximum at regular diffraction angles that are determined by the pixel spacing. The dominant diffraction order changes as the interface angle is changed. When the interface angle is set to concentrate the beam energy at one diffraction maximum, then the efficiency can be quite high. Once the interface angle departs from this value, energy is quickly distributed to other diffraction orders. Most of the side lobes are more than $10 \mathrm{~dB}$ lower than the peak value. Figure 3-6 is another plot of the far-field diffraction angle and interface apex angle dependence in normalized scale.

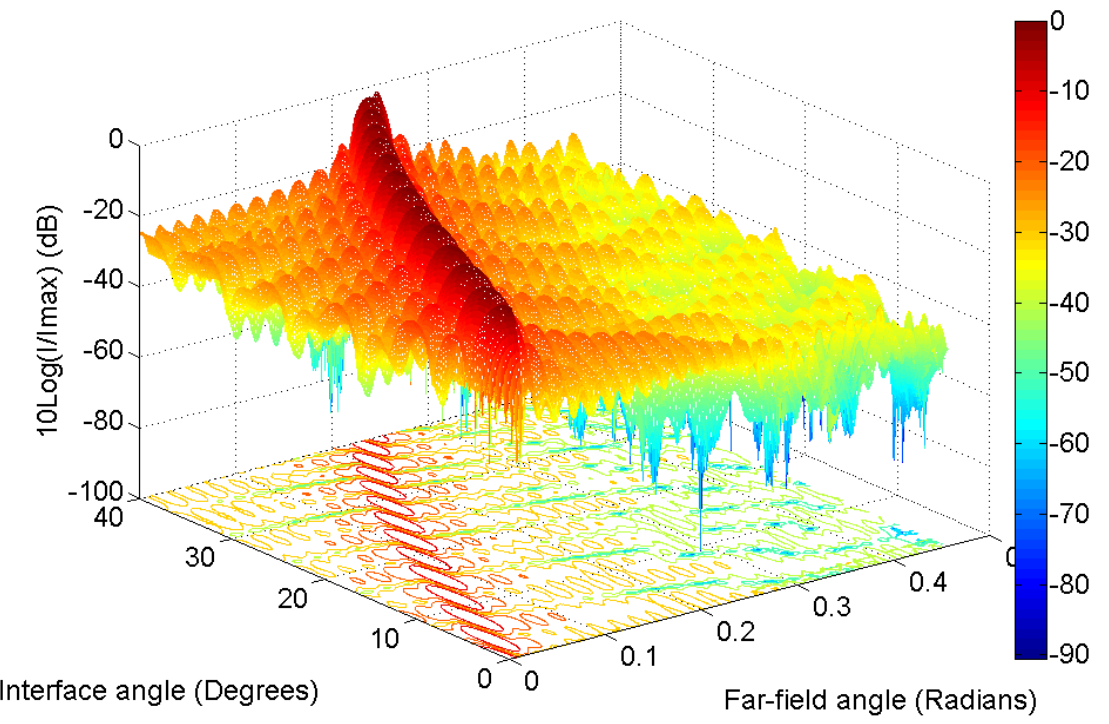

Figure 3-5: 3D mesh plot with contour, $x$-axis is the far-field angle and $y$-axis is the interface angle of the 2 liquids and $z$-axis is the logarithm of normalized intensity at corresponding far-field angle. 


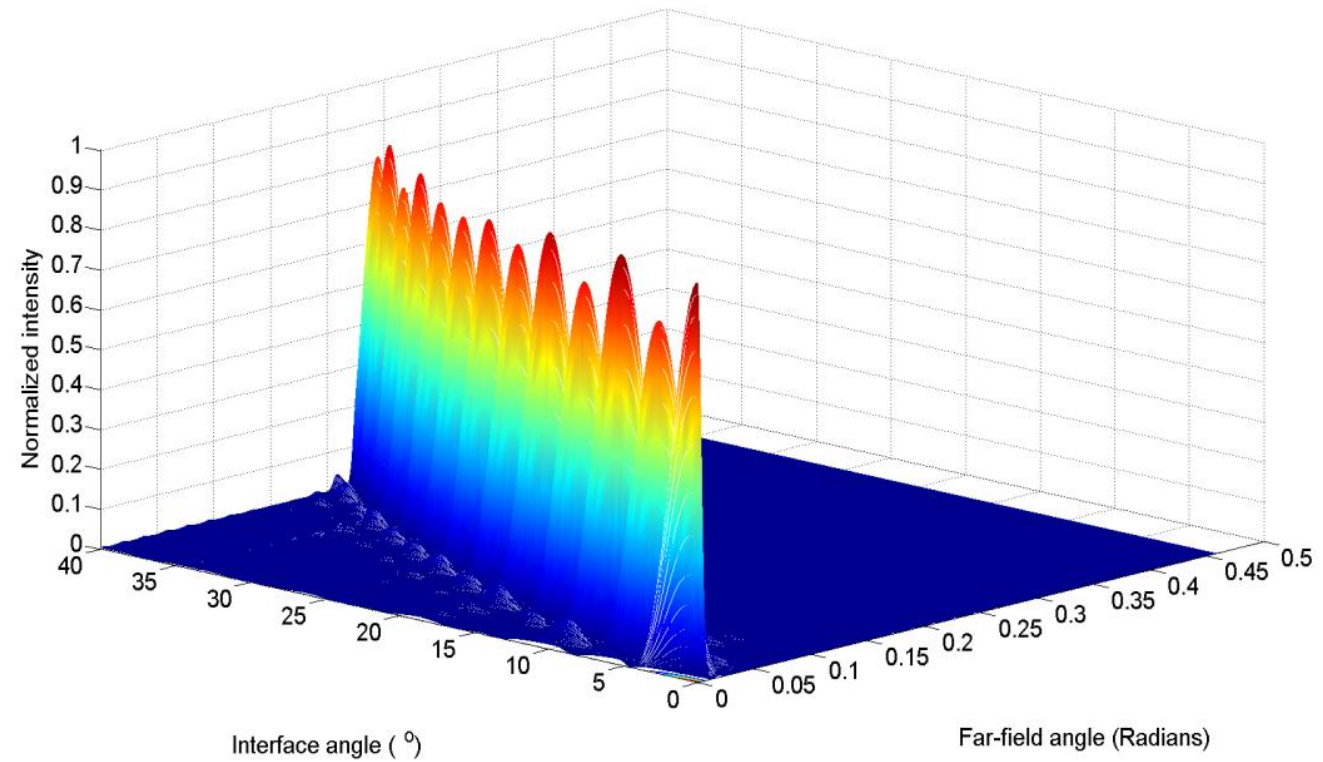

Figure 3-6: Far field angle and interface angle dependence for single-prism EMP arrays

From Figure 3-5 and Figure 3-6 we observed that only at certain interface angles the efficiency achieves a local maximum. As the interface angles increases, the far-field energy is transferred among several main lobes where a main lobe goes down and a side lobe goes up and eventually takes over the main lobe but these lobes remain still in far-field, which gives rise to the discrete far-field angular coverage. Low efficiency is primarily due to the phase mismatch between neighboring EMP pixels.

Phase mismatch between neighboring pixels is a key factor besides the reflectivity that accounts for the energy being redirected to multiple diffraction orders in the far-field. The phase change across each pixel can be estimated as follows:

$$
\Delta \varphi=2 \pi / \lambda \cdot \Delta n L \tan \theta
$$

Where $\Delta n$ is the refractive index difference between the two liquids, L as the length of the EMP unit cell, and $\theta$ is the interface angle. Theoretically we would get above $90 \%$ 
efficiency (determined by the fill factor, reflectivity of sidewall) if the phase change across a pixel is a multiple of $2 \pi$. Any deviation from the ideal values is a phase mismatch that contributes to side lobe competition in the far-field, thus loss of beam steering efficiency.

With the phase match analysis above, we can go back to Figure 3-5 and 3-6, the 3D logarithmic plot of far-field intensity versus interface angle and far-field diffraction angle. The diffracted intensity has a strong dependence on the interface angle due to the phase matching conditions mentioned above. The far-field diffraction angles are fixed and the intensity distributed among various diffraction angles are shown for the one-dimensional case. The bottom plane of the figure is a contour plot of the logarithmic intensity for better viewing of the beam steering efficiency. The dominant diffraction order shifts among locally adjacent lobes as the interface angle is changed; the maximum of a particular diffraction order corresponds to the phase matching condition in Eq. (16). The discrete diffraction lobes appear due to the fact that the EMPs are periodically arrayed in the near-field domain, which makes discretization in far-field angular pattern a natural consequence.

To numerically determine the 1D efficiency in the far-field, an algorithm is introduced here. First we start by finding the global maximum of the far-field irradiance. Then we find the indices (far-field angles) of local maxima and minima of the irradiance. At this time we are able to find the two adjacent local minima to the global peak, which contains the highest power and defines the so-called main lobe in the far-field. The efficiency is given by the ratio of the power in the main lobe to the total power. This efficiency calculation 
result is shown in Figure 3-7. In this plot, the incident Gaussian beam is deflected by 20 pixel EMP arrays. The interface angle is continuously changed from 0 to 40 degrees and the efficiency is plotted with respect to the discrete far-field angle.

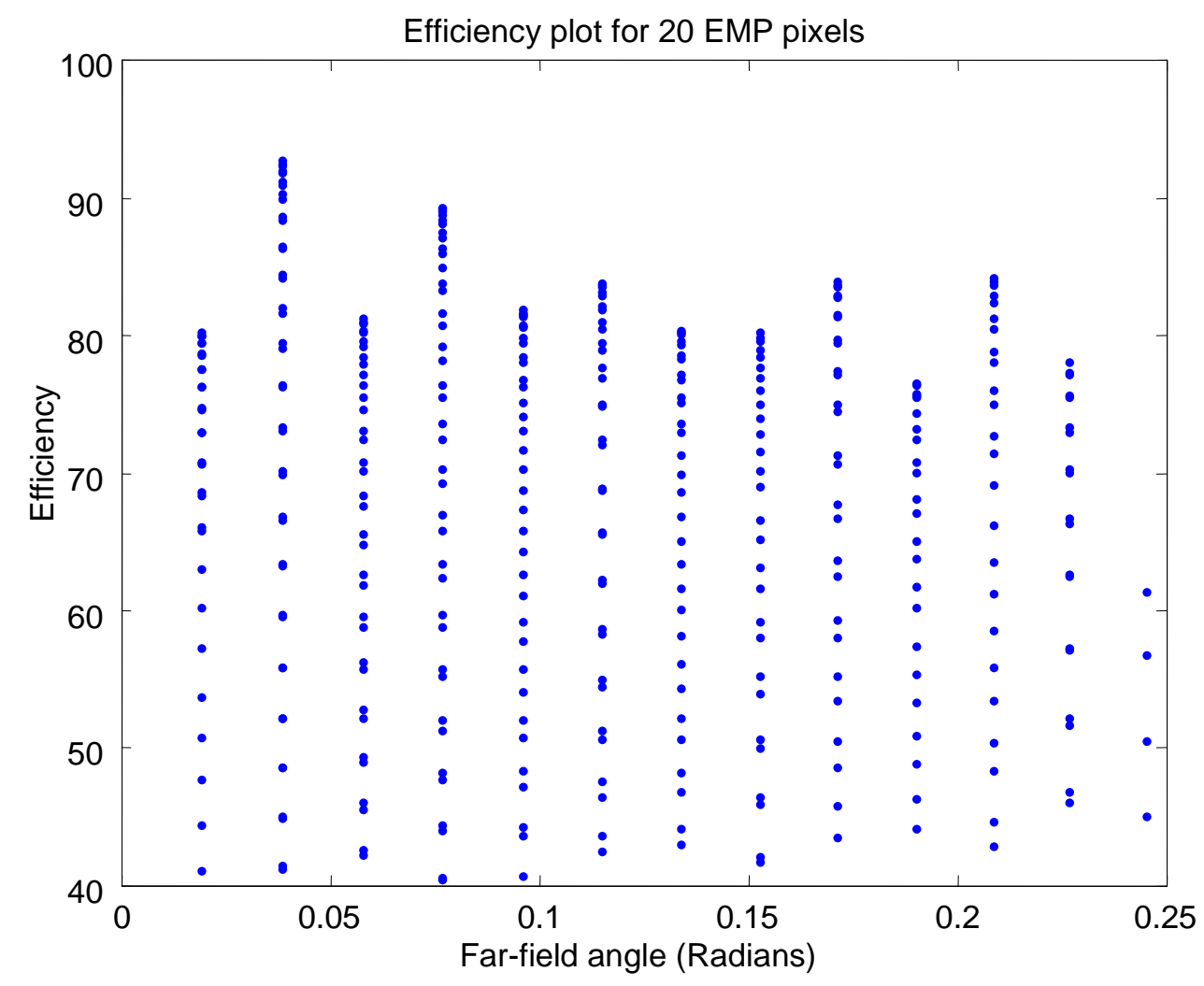

Figure 3-7: Efficiency plot of 1D single-prism EMP arrays.

The comparison of predicted local maximum efficiency based on Eq. (16) and simulation-found local maximum efficiency with respect to interface angle is shown in Figure 3-8. The curve is draw to show the geometrical effect of the far-field angle; it passes through all the points labeled "theory". The discrepancy in the angle between the theory and simulation points is due to other issues such as reflectivity and fill factor that are not accounted for in Eq. (16). As the interface angle increases, the accumulated phase difference grows at a constant rate. The line clearly and quantitatively captures the trend in 
the simulations.

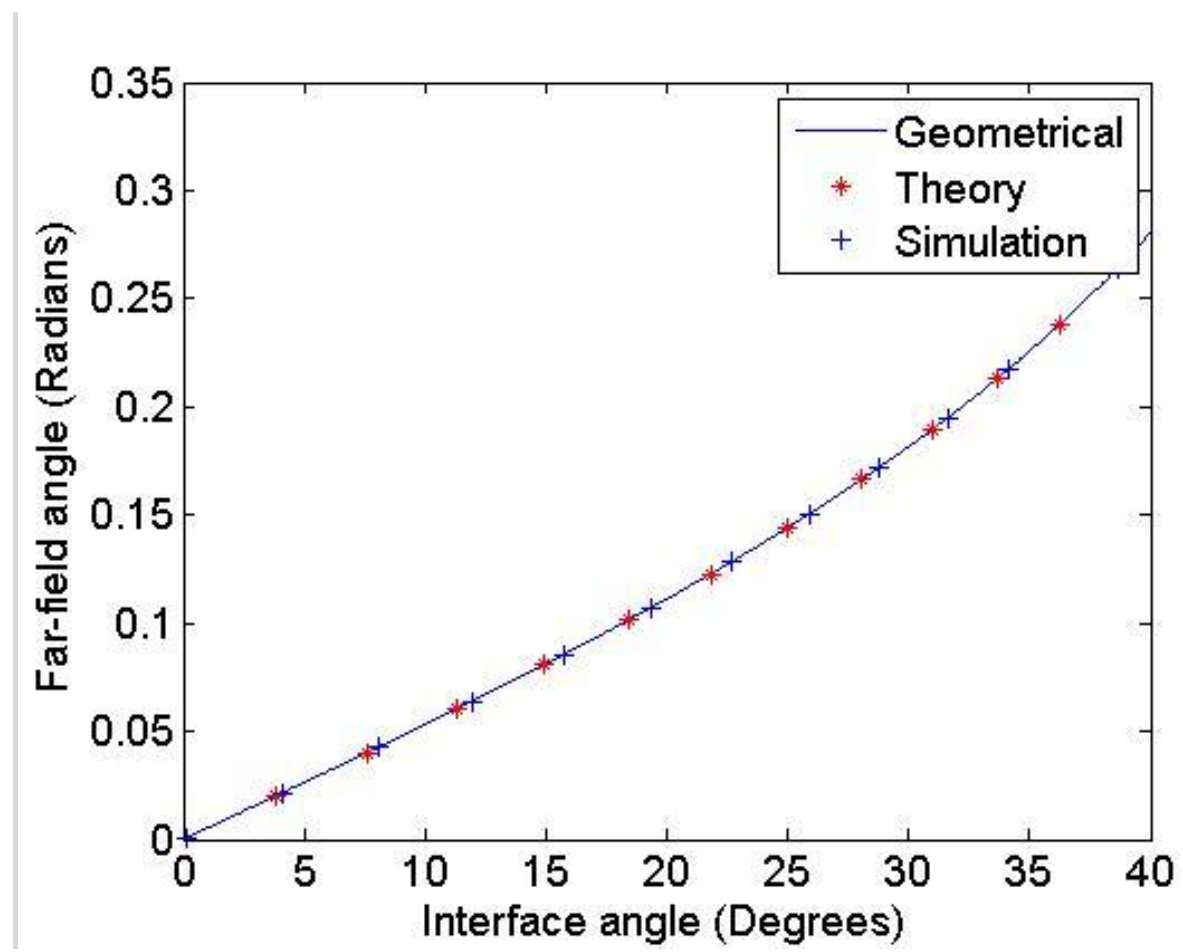

Figure 3-8: Comparison of predicted maxima based on Eq. (16) and simulation results based on beam propagation algorithm

\subsubsection{PHASE TILT OF INCIDENT BEAM}

Based on the above discussion, there is a straightforward solution to enhancing the steering efficiency and filling in the far-field angles continuously between the diffraction maxima by introducing a small tilt angle on the incident beam as depicted in Figure 3-9. The tilt angle is imposed on the incident Gaussian beam by adding a changeable phase tilt term across the Gaussian incident beam function before it enters the EMP array. The tilt can be zero where the diffraction maxima occur, i.e. the interface angles where we already achieve the highest efficiencies. Then we add small tilt angles on the incident beam to fully 
cover the far-field angles between two adjacent diffraction maxima to maintain a high diffraction efficiency over all angles in that range. The fine tilt angles can be either positive or negative. The small tilt angles could be practically accomplished by adding a fine-tuning Optical Phased Array (OPA) immediately before of the first surface. Other solutions using a simpler phase adjustment under each pixel have been proposed [9].

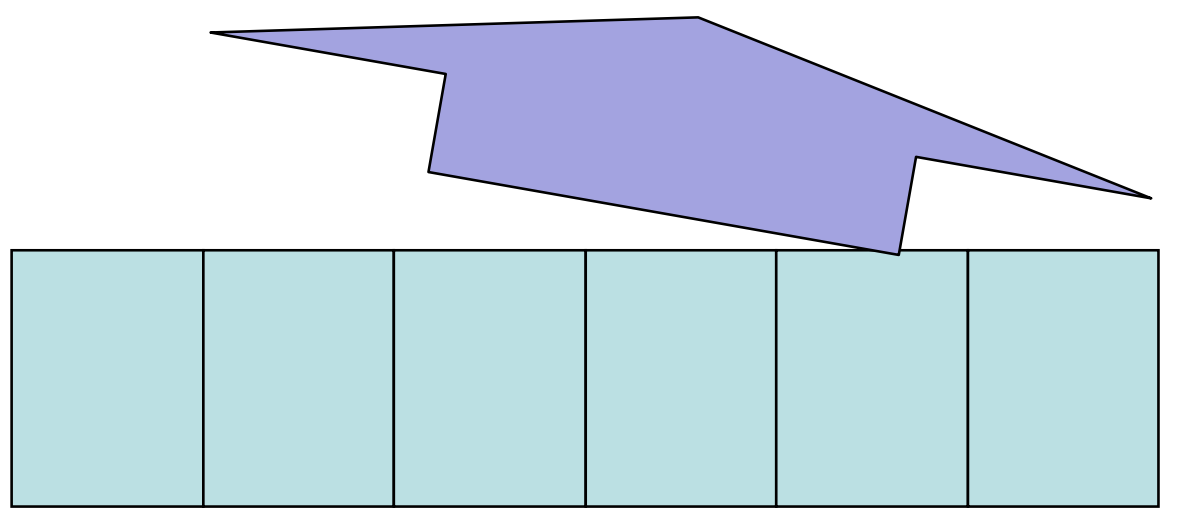

Beam tilt wedge

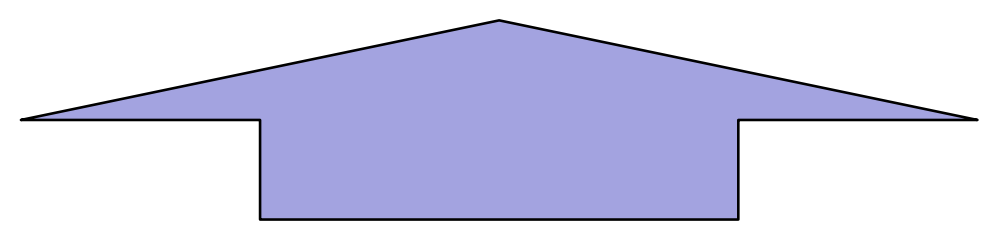

Figure 3-9: Introduction of tilt in front of EMP arrays.

Figure 3-10 is the far-field efficiency plotted with both input beam tilt angle from local maximum that slightly deflects the incident beam to the right and the left with tilt increment of 0.1 degree. This is refined with increment of 0.05 degree shown in Figure 3-11. In order to eliminate the discontinuity in efficiency, we redo the efficiency calculation with tilt increment of 0.05 degree only from higher peaks in Figure 3-11. The result is demonstrated in Figure 3-12. A tilt angle of up to $1^{\circ}$ was used in our calculations 
and we achieve far-field diffraction efficiency maxima higher than $80 \%$ for up to $12^{\circ}$ far-field deflection angle. This enables a continuous beam steering capability for the input beam. The separation between the orders for our EMP spacing is about 0.05 radians, which is about double the required fine angle steering angle, since only a correction of half the separation is required.

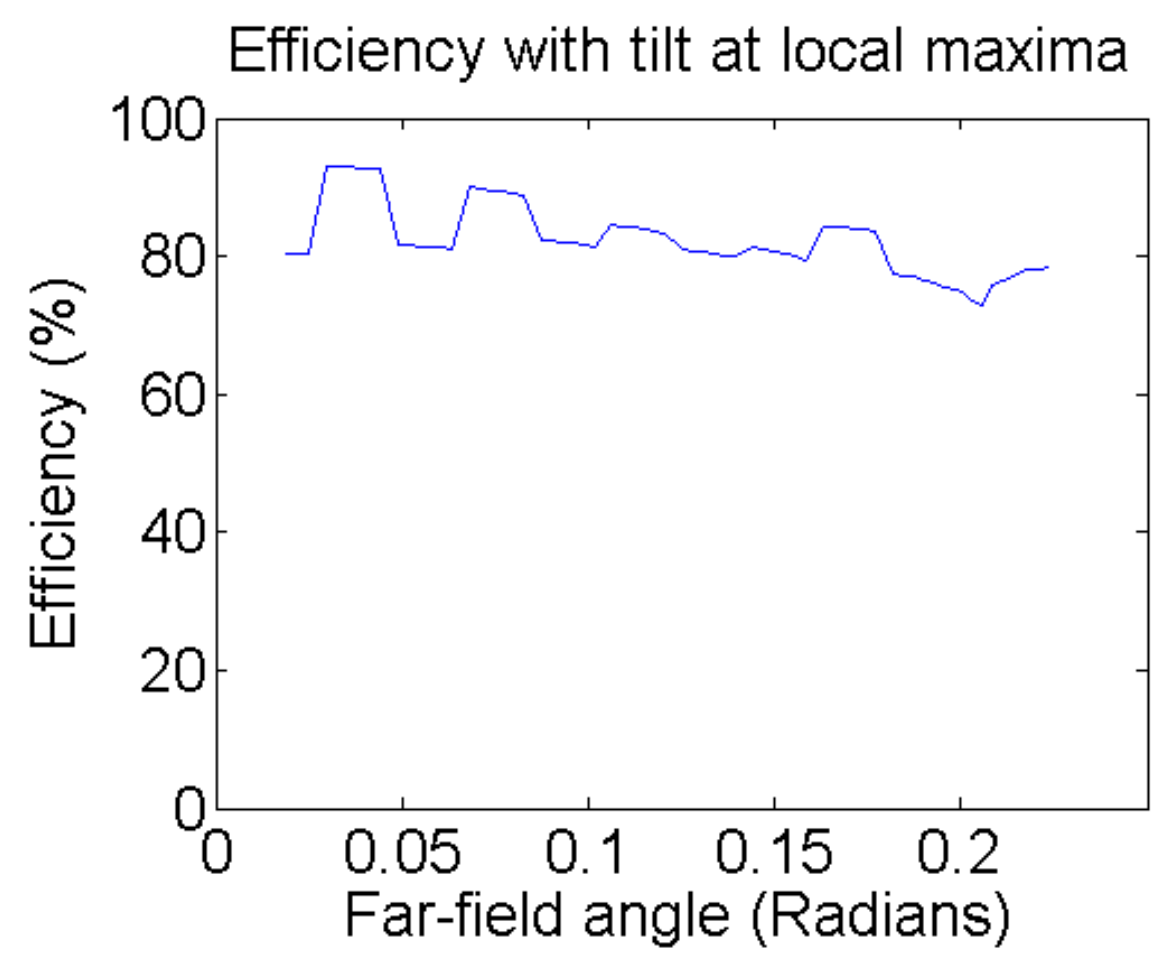

Figure 3-10: Efficiency plot from local maxima with tilt increment of 0.1 degree. 


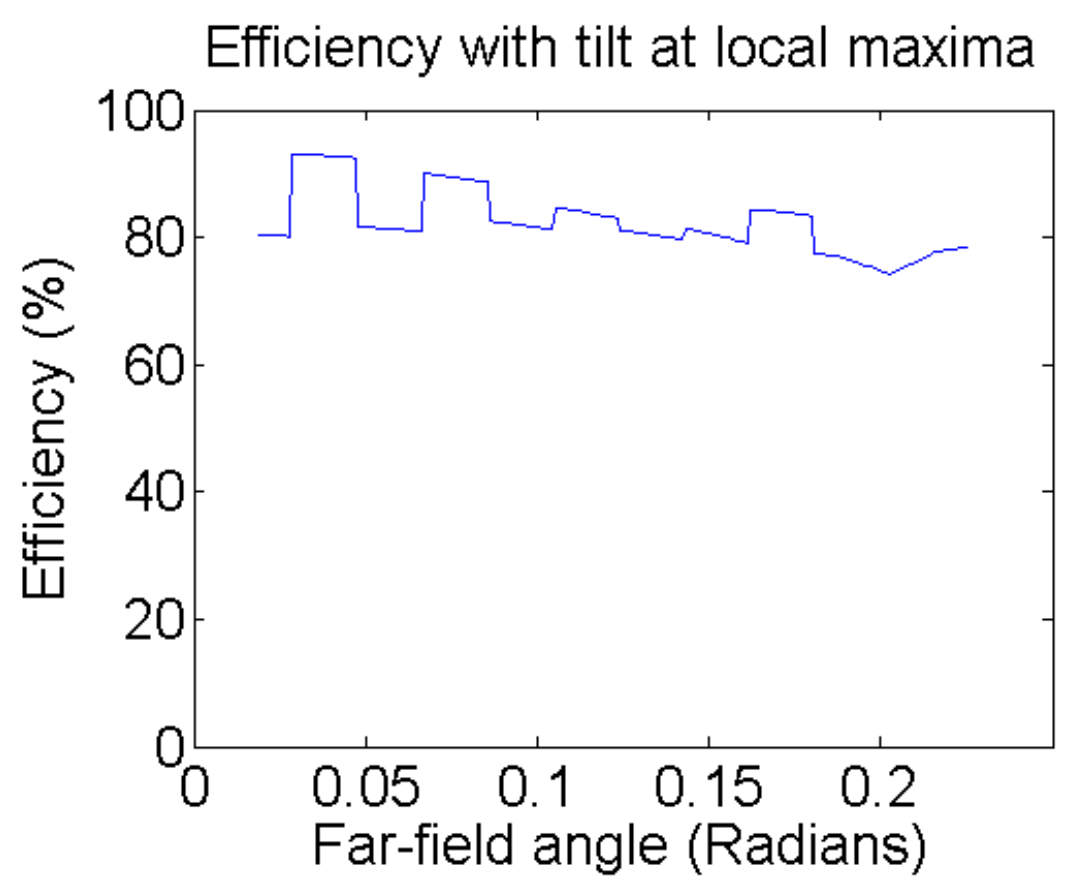

Figure 3-11: Efficiency plot from local maxima with tilt increment of 0.05 degree.

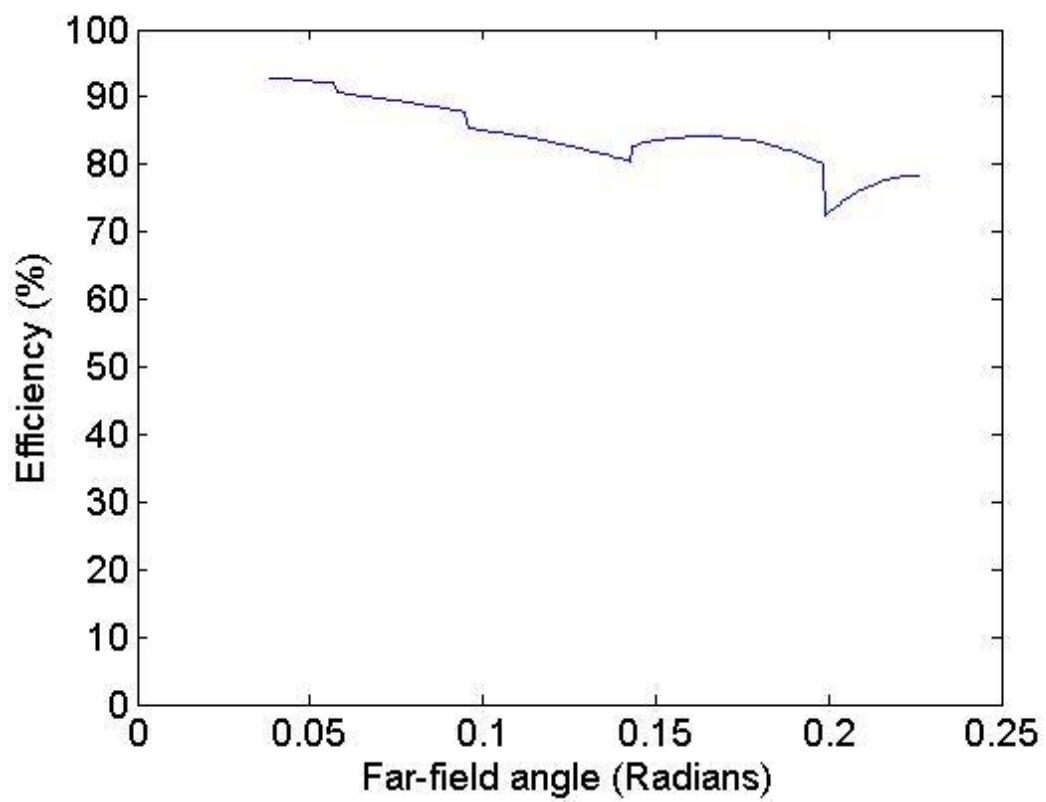

Figure 3-12: Optimized efficiency plot with tilt from higher peaks for 1D single-prism EMP arrays.

\subsubsection{D SINGLE-PRISM EMP ARRAYS}

Based on 1D modeling, we extend the single-prism EMP arrays to two-dimensional pixel structures. A 3D view of a $2 \times 2$ structure is shown in Figure 2-3 in Chapter 2. In the 
2D EMP array case, two interface angles of intersecting planes are adjustable. By keeping aqueous-oil interface lines at two opposite walls parallel, two dimensional beam steering is possible.

We first consider the case where the two interface angles $\theta_{x}$ and $\theta_{y}$ are equal. In our simulations, a 10x10 EMP array is investigated. In Figure 3-13 we demonstrate a series of far-field and near-field intensity patterns starting from $\theta_{x}=\theta_{y}=5^{\circ}$ with an increment of 5 degrees and centered an incident Gaussian beam whose beam waist is 30 microns wide. In these figures the near-field intensity is plotted on a linear scale and the far-field on a logarithmic scale. The near-field beam is characterized by large fluctuations as noted previously for the 1D case. The sidewall effects are much more apparent here. The far-field intensity has one main diffraction lobe with smooth peaked structures distributed over several diffraction angles. The intensity was cut off below $-100 \mathrm{~dB}$ for clarity in this figure. The energy is broadly distributed over regions close to the axis and has less energy between the two major axes.

$$
\theta_{x}=\theta_{y}=5^{\circ}
$$



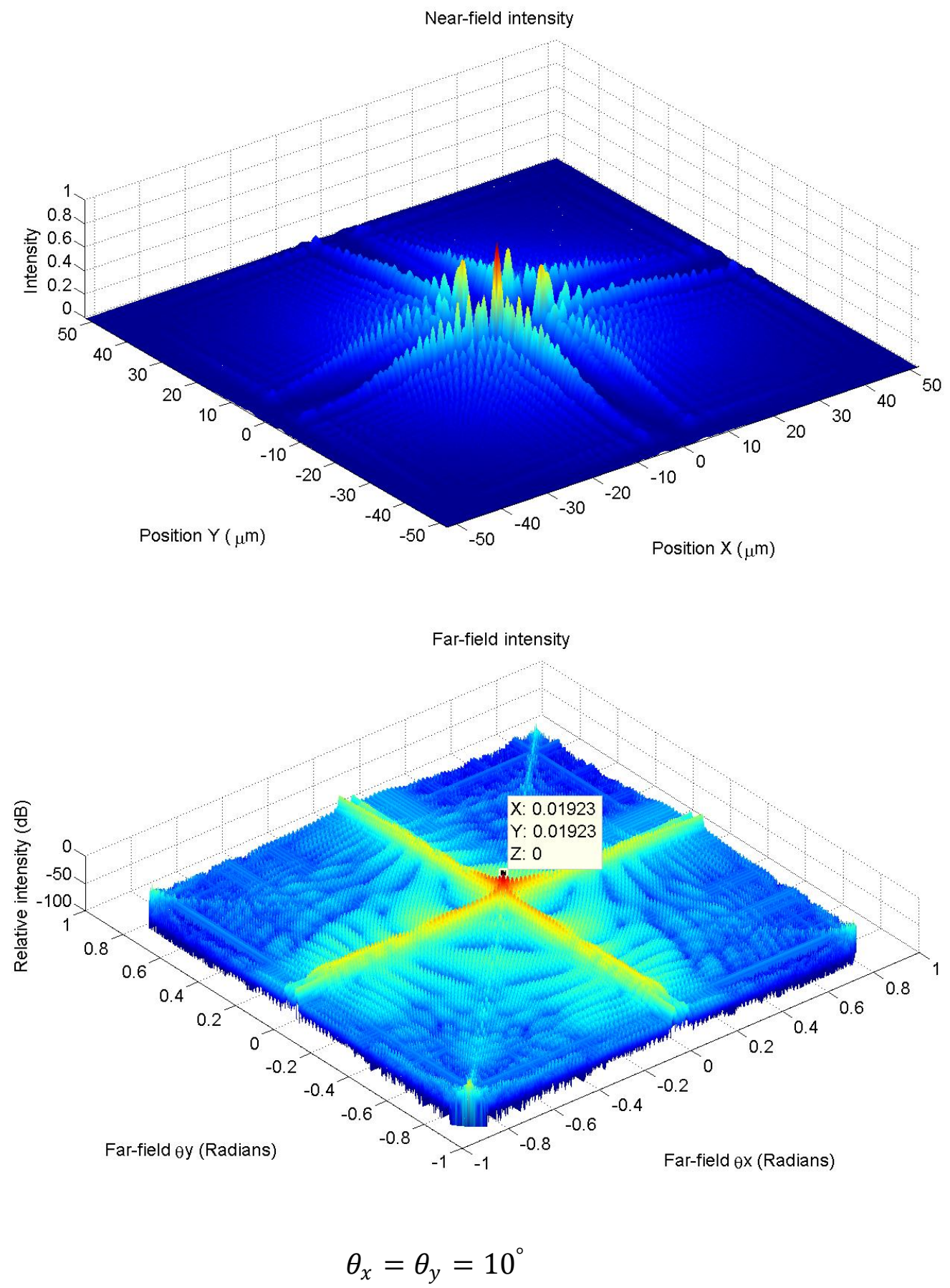

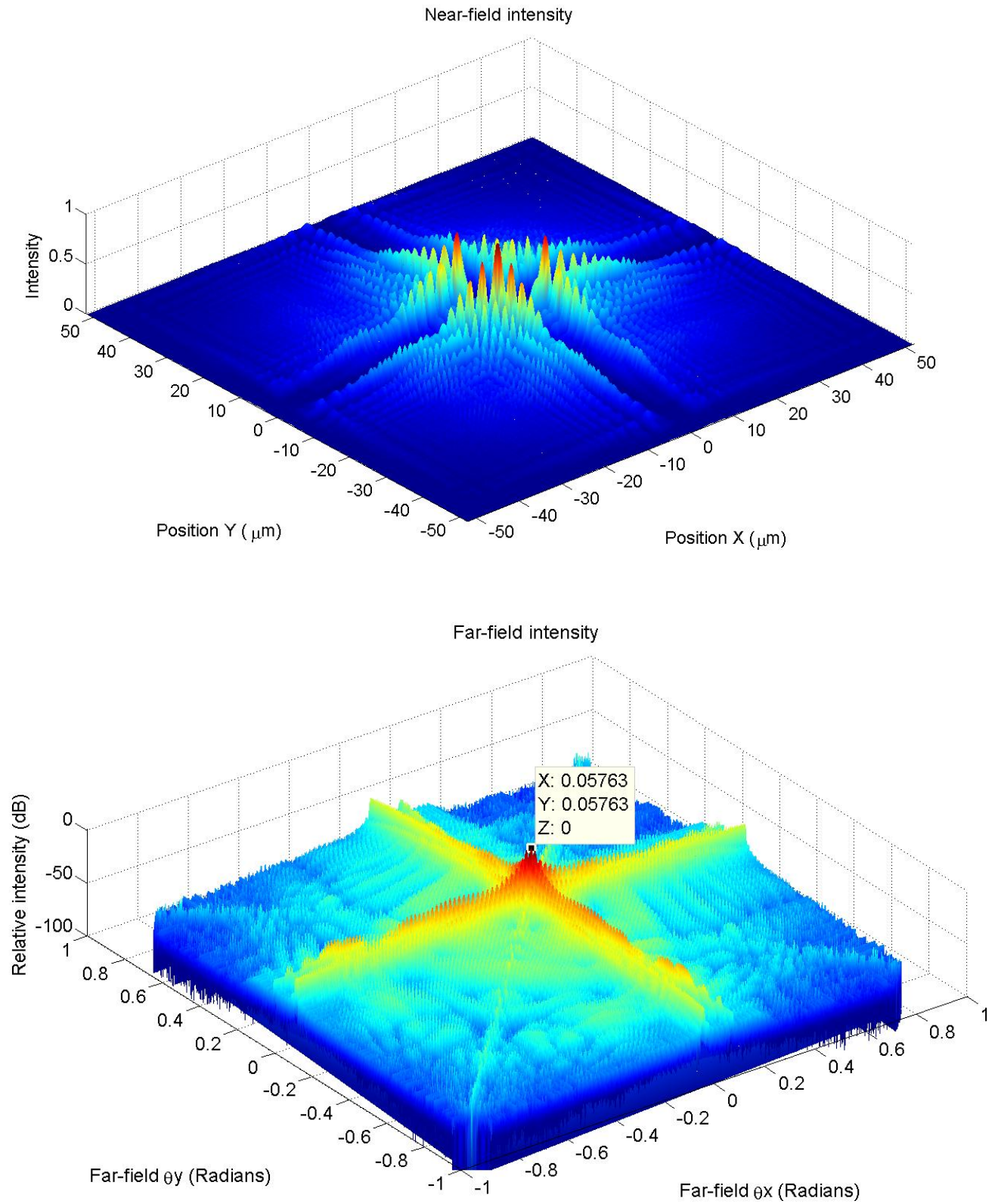

$$
\theta_{x}=\theta_{y}=15^{\circ}
$$



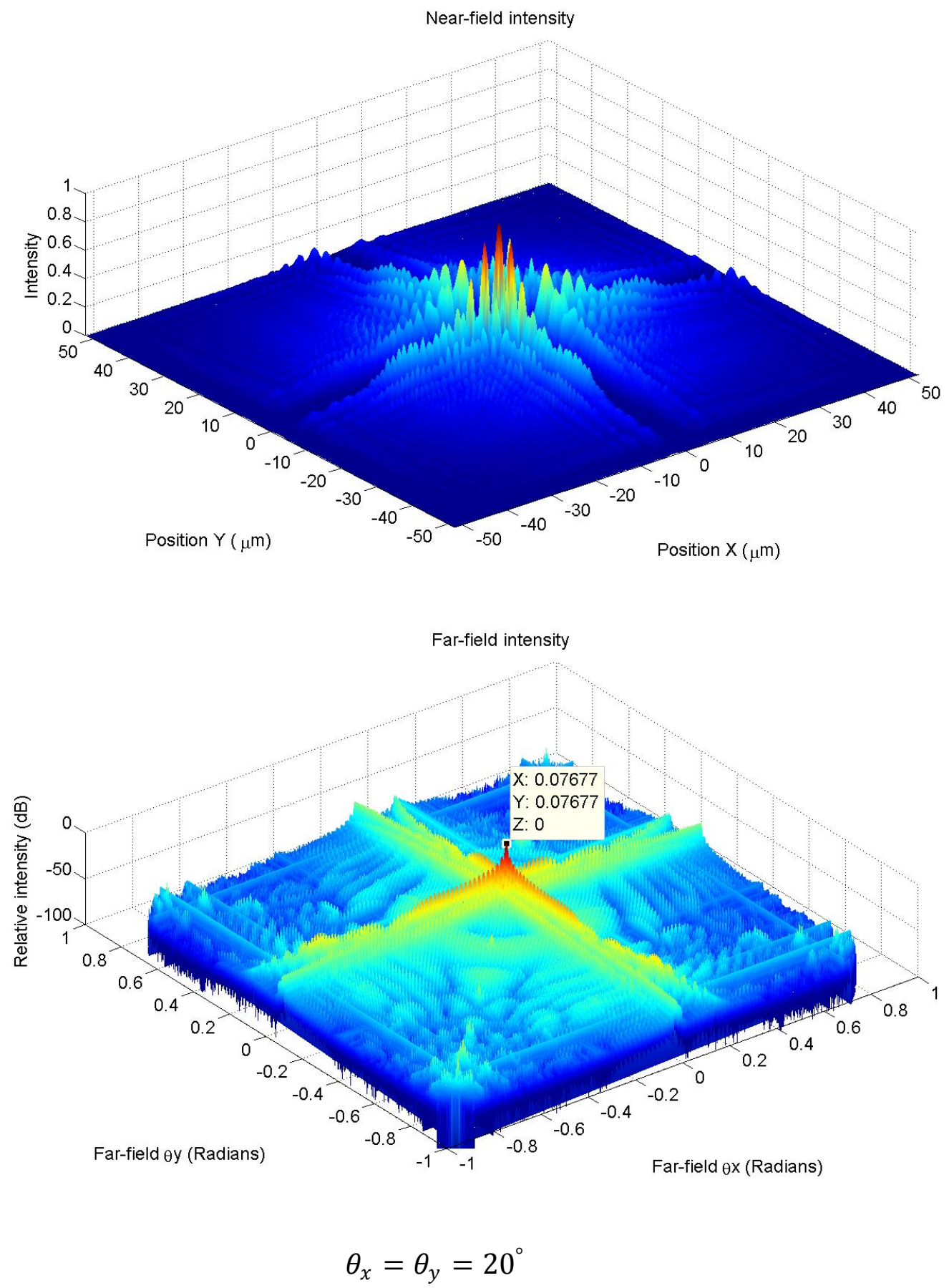

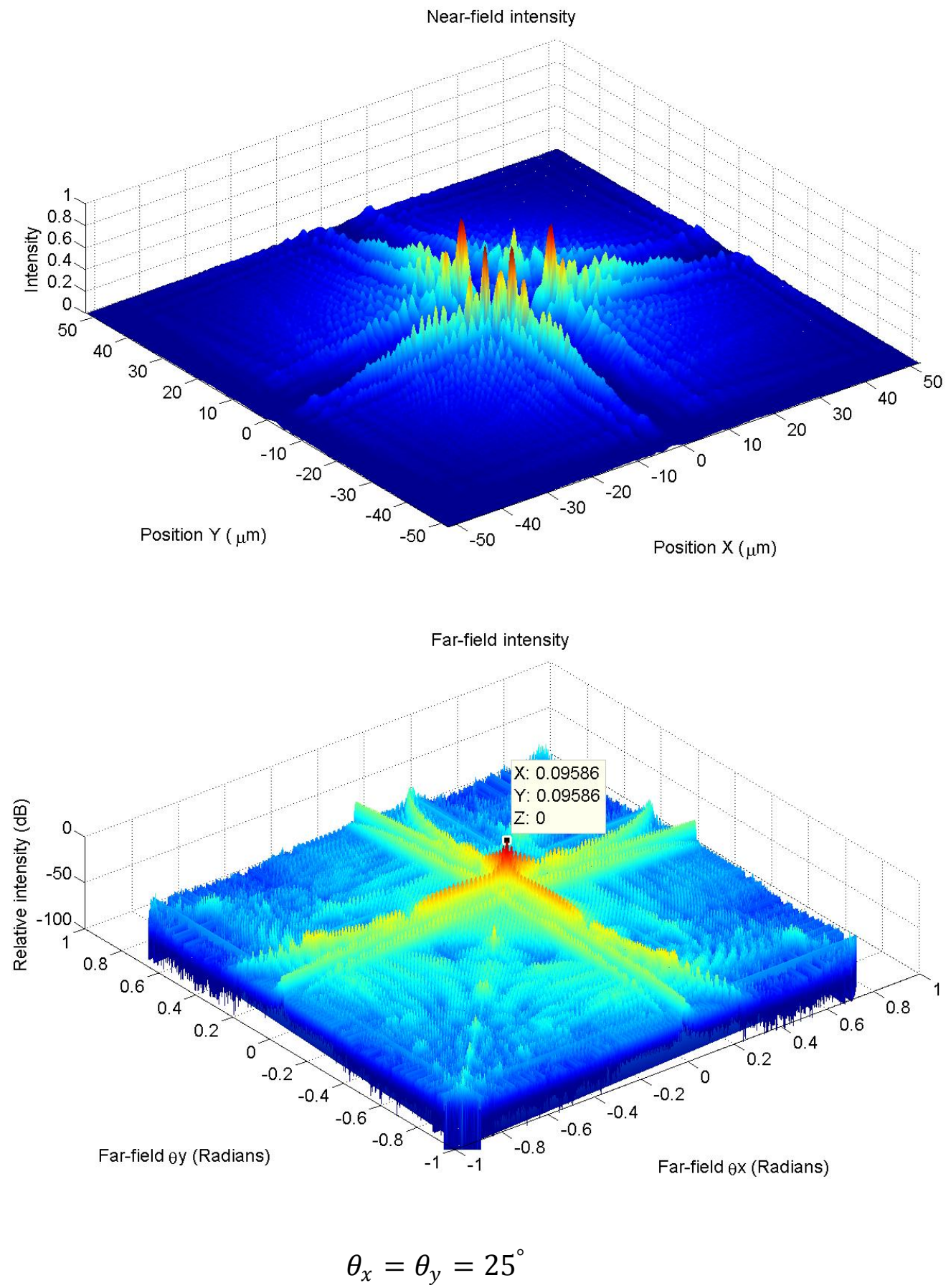

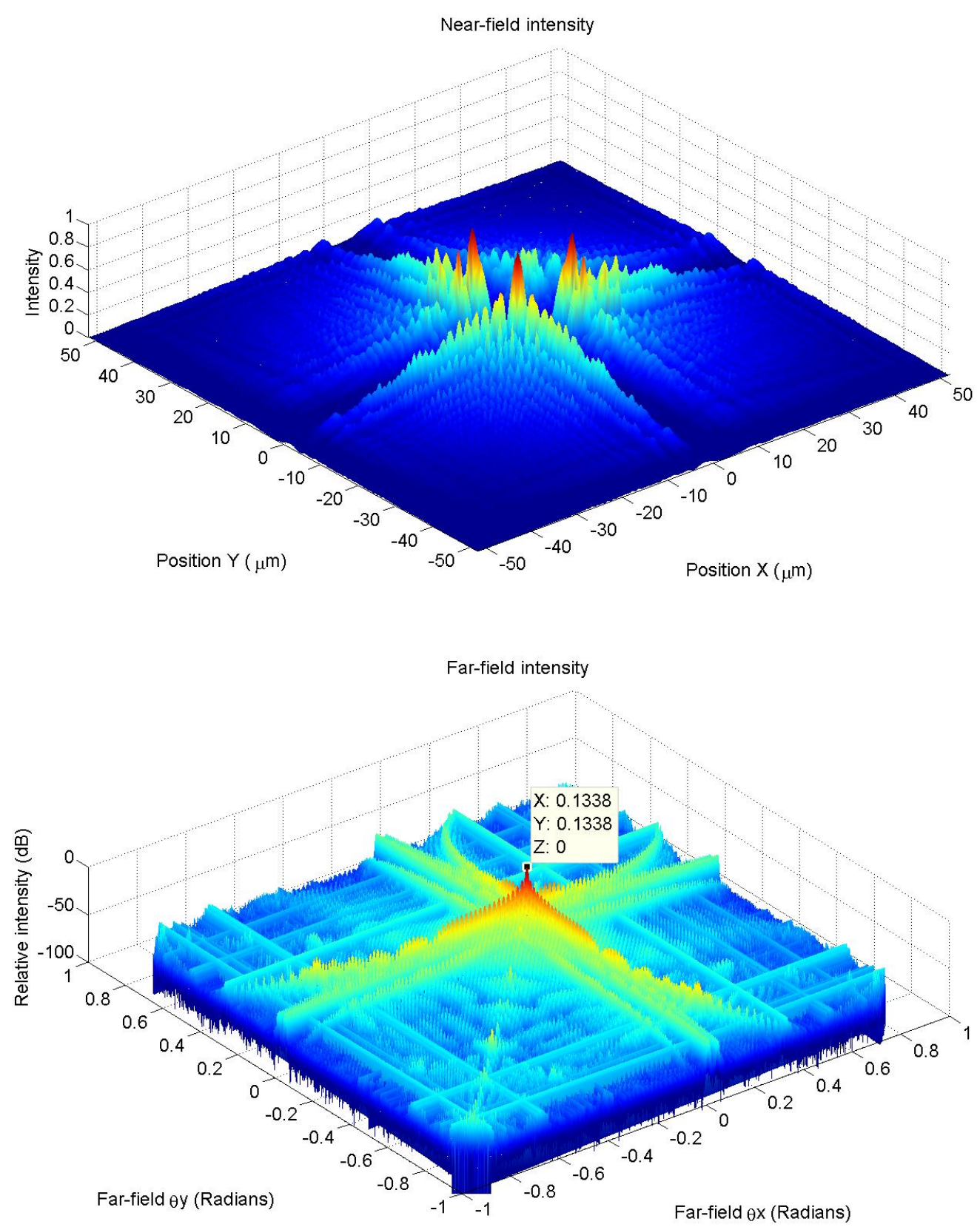

Figure 3-13: Near-field and far-field intensity for 10x10 2D single-prism EMP Arrays for various interface angles.

A 2D EMP steering efficiency algorithm is introduced to calculate the far-field efficiency for the 2D case. A scan is performed to find an intensity maximum then we look for the two-dimensional region where the intensity is greater than 0.368 , i.e. $\mathrm{e}^{-1}$, of the maximum, in the two-dimensional domain. This step is shown in Figure 3-14, where we 
have $\theta_{x}=\theta_{y}=20^{\circ}$. Assuming the minimum distance from the peak (center) to the edge of scanned region to be the radius, we double the radius to define our main lobe radius, in which case the intensity drops to 0.0183 ; we used this procedure as a reasonable threshold for main lobe detection as shown in Figure 3-15. All the irradiance that falls within main lobe radius will be add up to its diffracted energy. This procedure was used to calculate the diffraction efficiency in 2D case after dividing the diffracted energy by total energy.

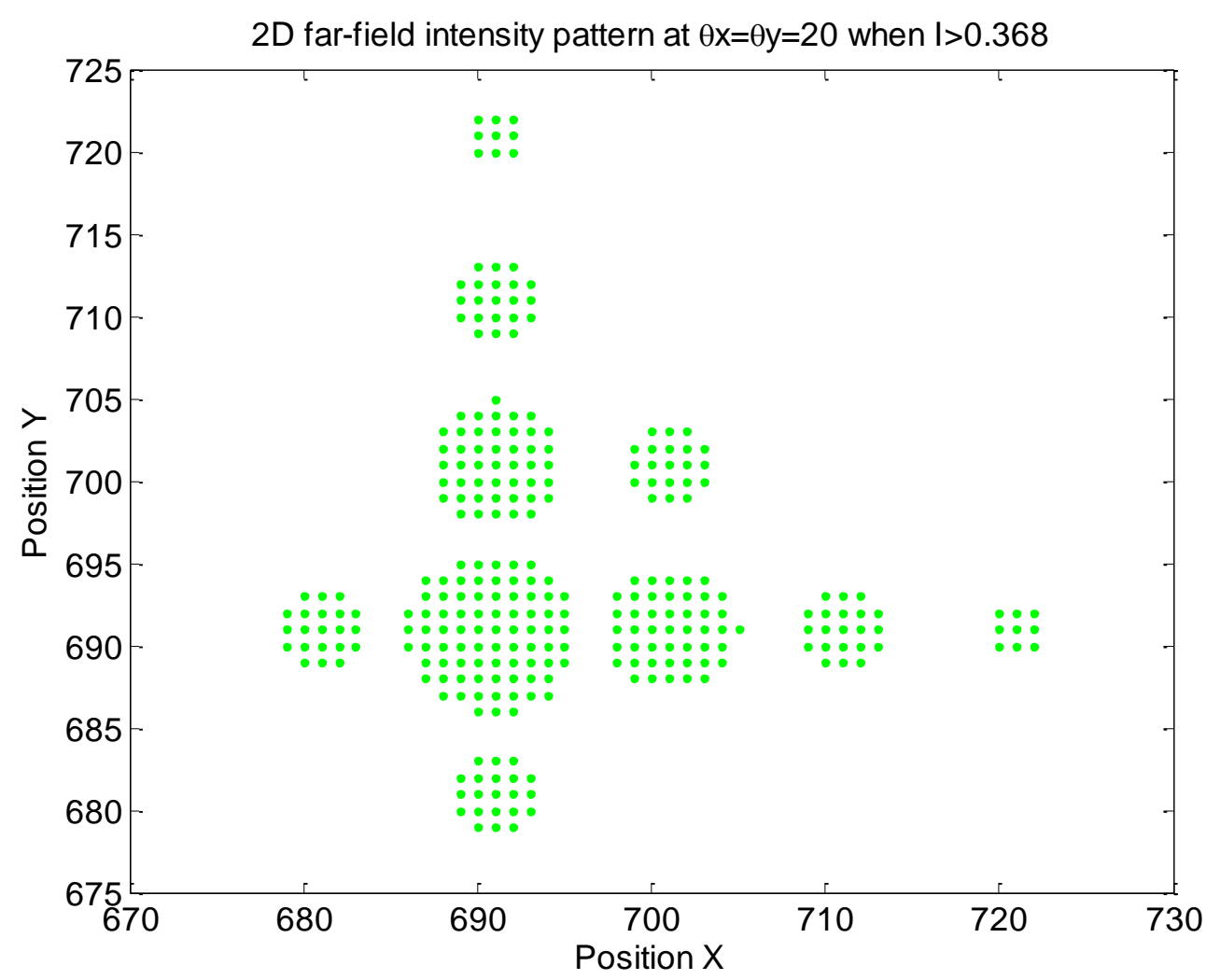

Figure 3-14: Far-field intensity pattern when I >0.368 for 10x10 2D single-prism EMP arrays. 


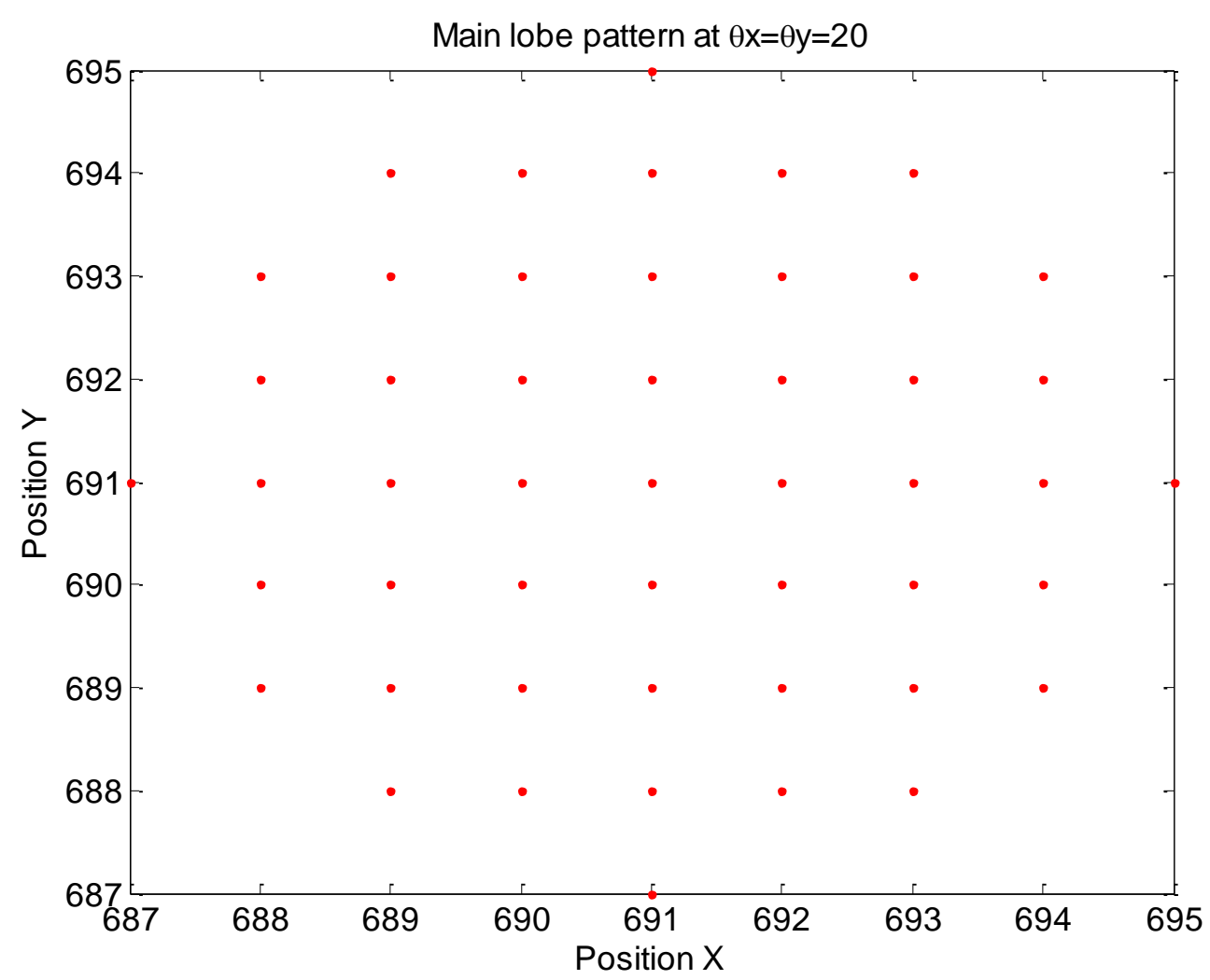

Figure 3-15: Main lobe detected using our method.

Beam steering efficiency is calculated using the algorithm discussed above for $2 \mathrm{D}$ EMP arrays as interface angles increase. Similarly to $1 \mathrm{D}$ case, the plot is discrete due to energy shifting among several lobes. Figure 3-16 is a plot of calculated efficiency and interface angle dependence. The efficiency is calculated as discussed previously by using the dominant diffraction maximum. As the interface angle changes to a mismatched phase angle the efficiency drops and energy is distributed from one peak to other diffraction peaks. The neighboring diffraction angle eventually becomes the next maximum and its efficiency rises until the optimal phase matching is again achieved. Figure 3-17 illustrates the efficiency evolution as both interface angles equally increase for 2D single-prism EMP arrays. To correct the undesirable loss of efficiency between the discrete diffraction angles 
a phase tilt also can be applied to the front of 2D EMP arrays. The tilt improves the efficiency between the far-field diffraction orders, as shown in Figure 3-18. The efficiency is maintained above $70 \%$ up to 0.1 radians in far-field. Higher far-field angles are achievable with modifications to the geometrical settings.

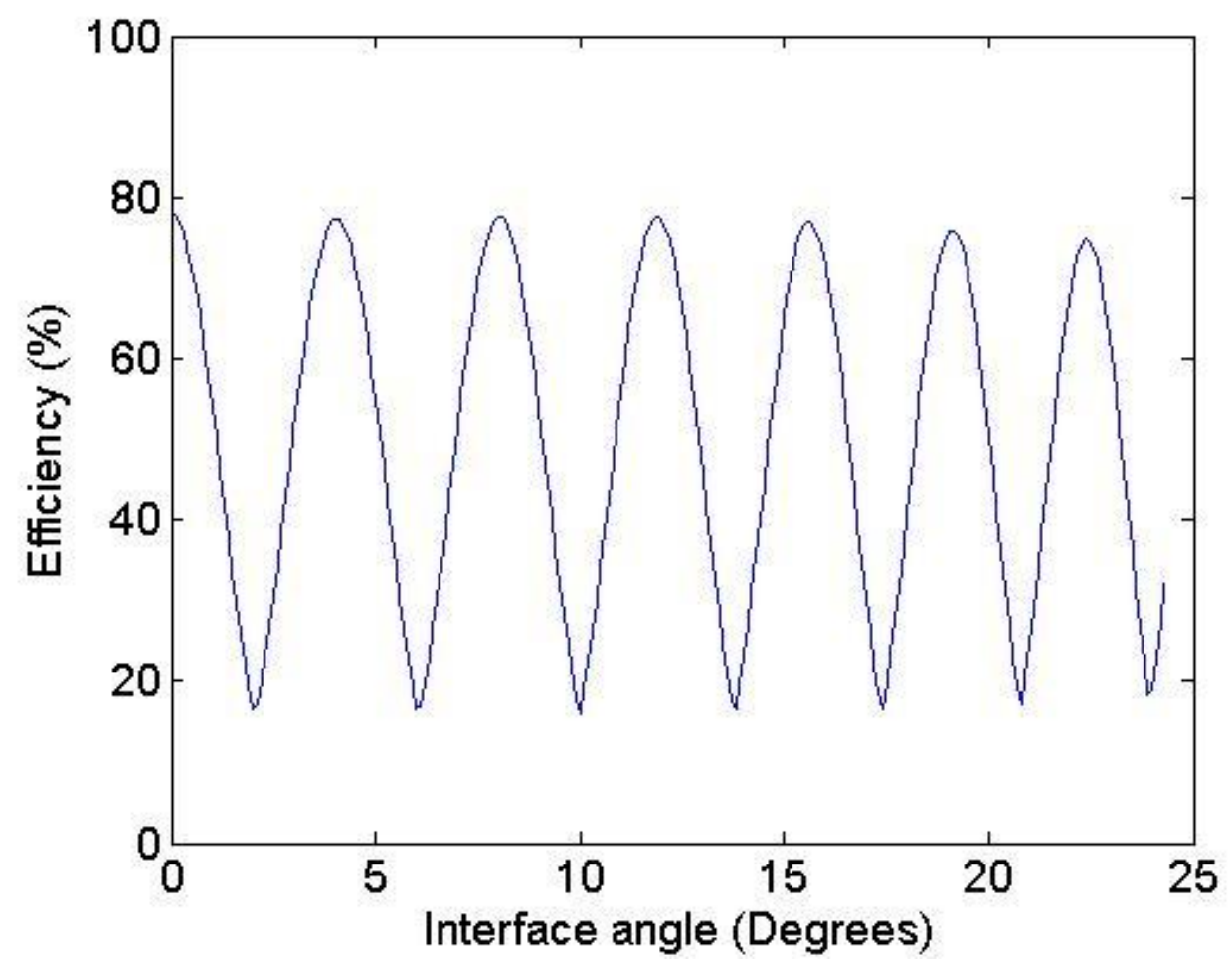

Figure 3-16: Calculated efficiency and interface angle dependence for 2D single-prism EMP arrays. 


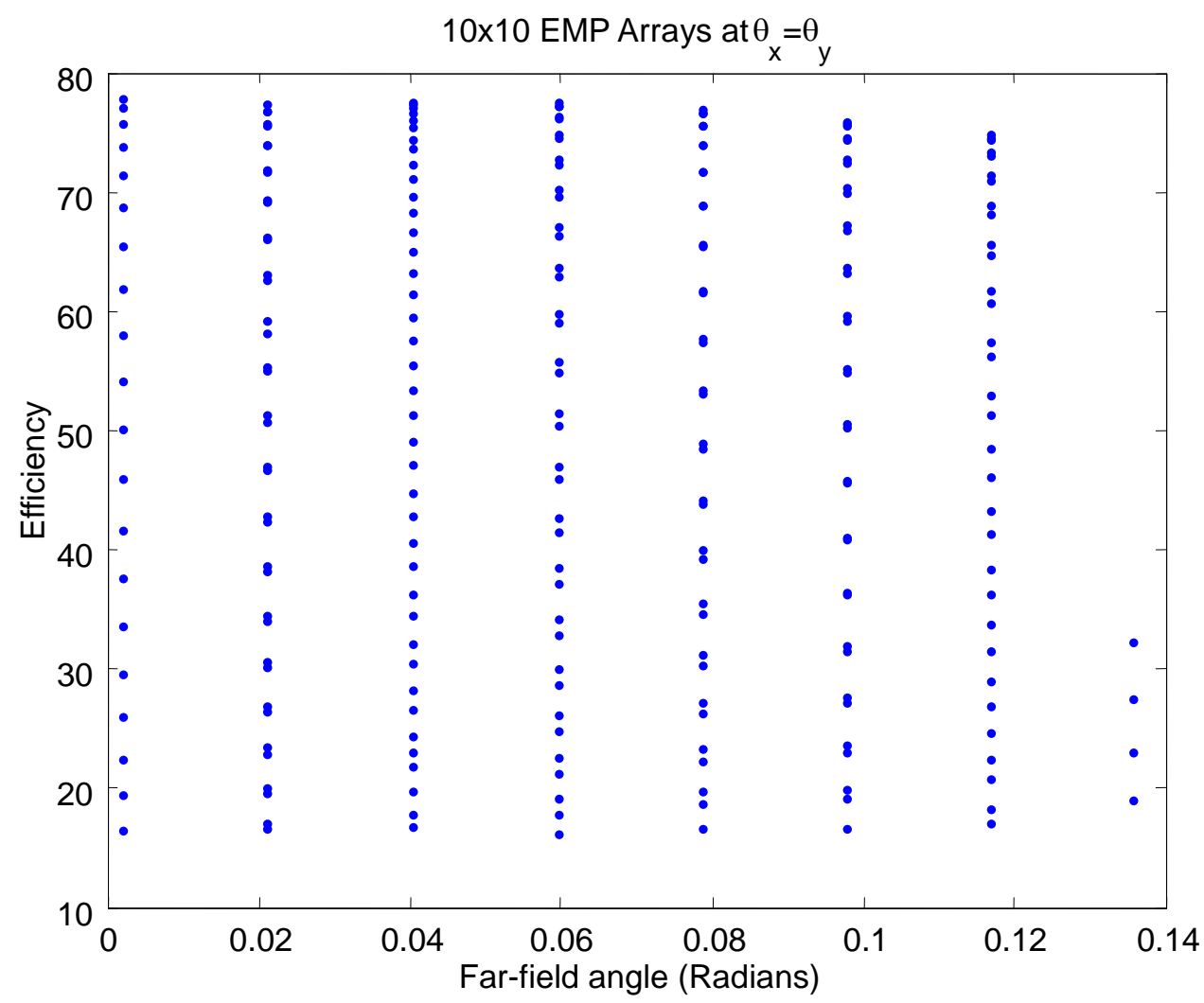

Figure 3-17: Far-field efficiency and interface angle dependence for 2D single-prism EMP arrays.

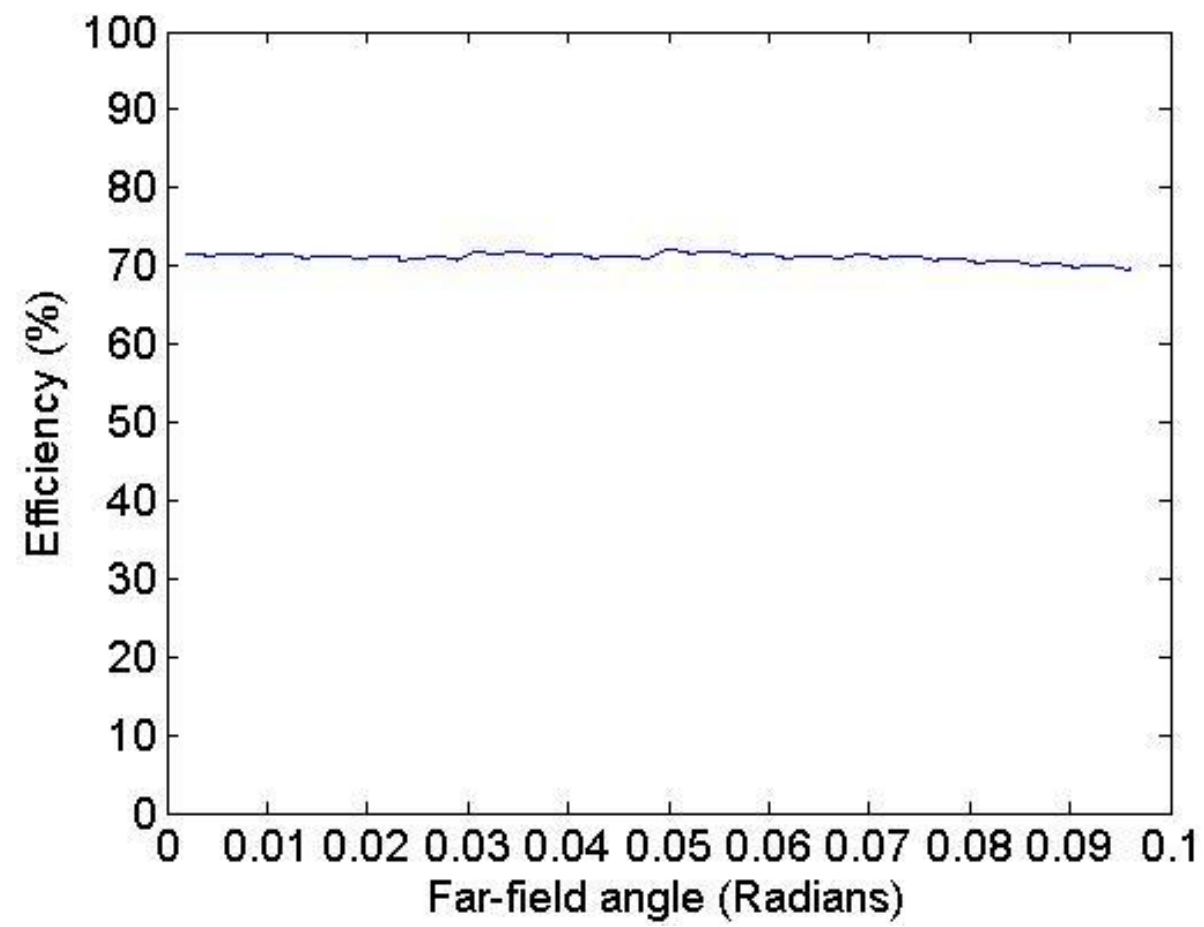

Figure 3-18: 2D EMP arrays efficiency with tilt for 2D single-prism EMP arrays. 


\subsection{BI-PRISM EMP ARRAYS}

\subsubsection{D BI-PRISM EMP ARRAYS}

A new design as shown in Figure 2-2 in Chapter 2 is previously introduced as a bi-prism structure where oil lies in the center of an EMP pixel sandwiched by water on top and below. The two oil-water interfaces can be controlled separately by placing two electrodes on one sidewall. This is physically achievable since on such a small scale, surface tension is dominant over gravitation effects. In this thesis, we will confine our discussion to the case where the two interface angles are equal.

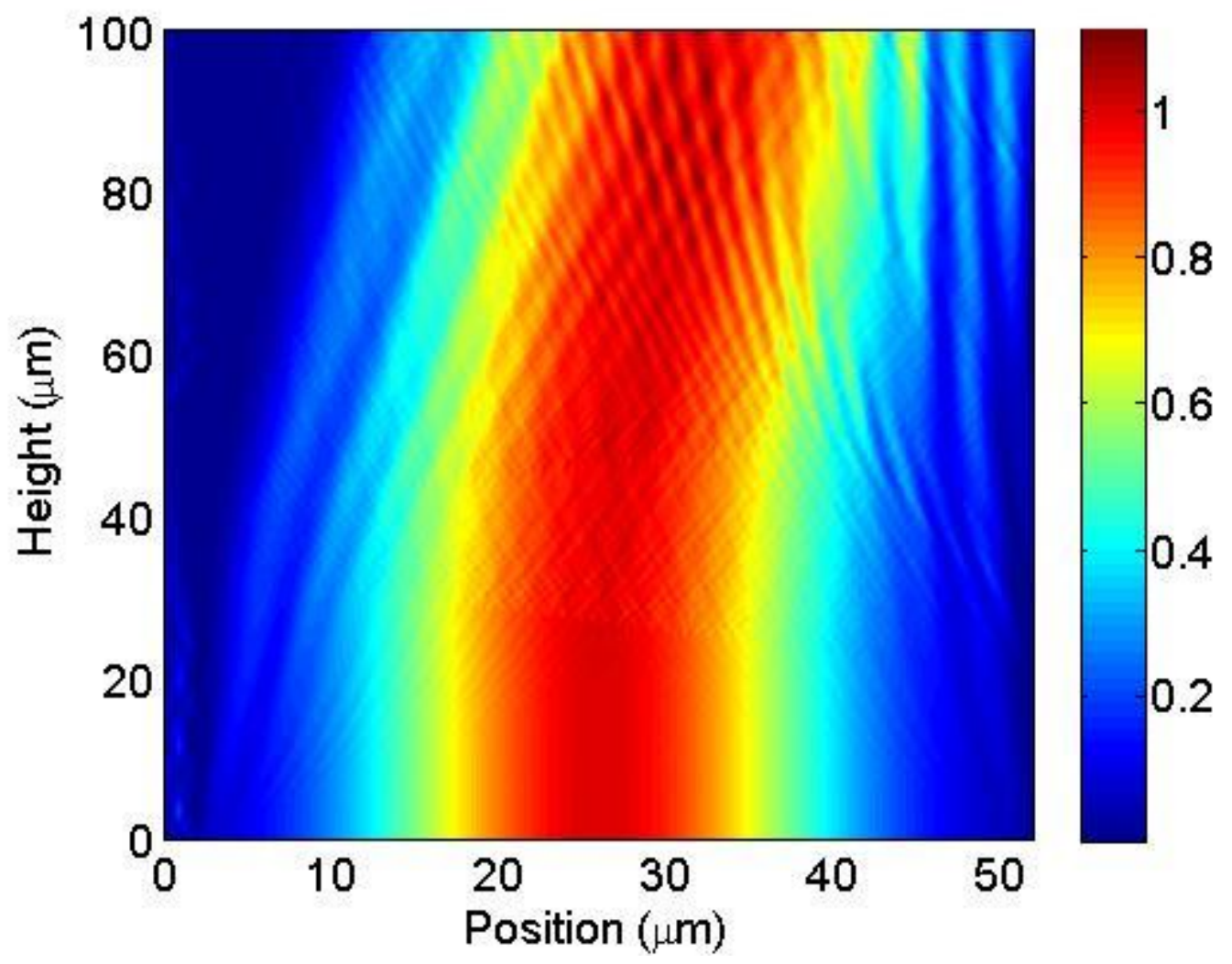

Figure 3-19: Intensity distribution inside a bi-prism EMP pixel at interface angle $\phi_{x}=\phi_{y}=15^{\circ}$. 


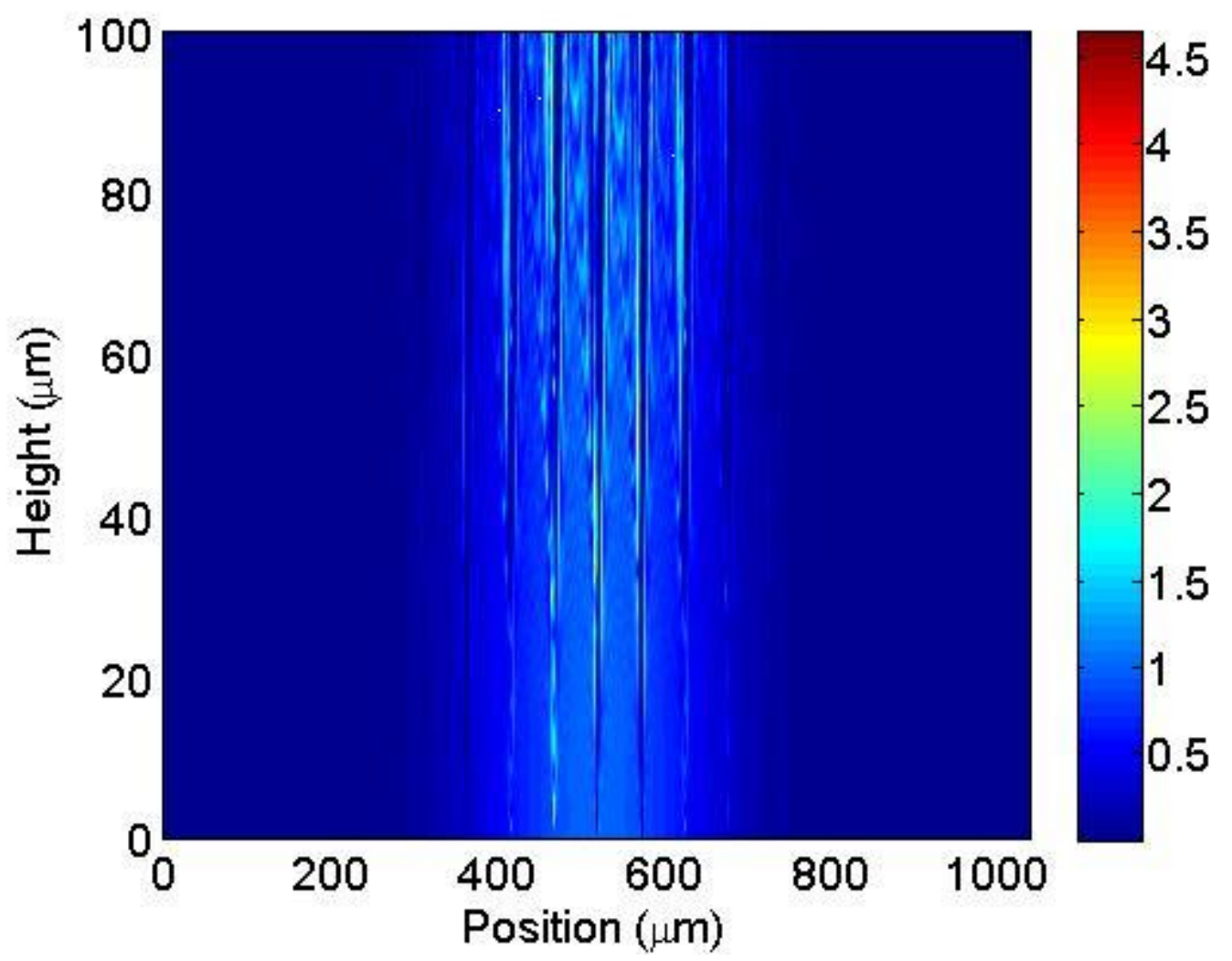

Figure 3-20: Intensity distribution inside 20 bi-prism pixels with beam waist of $200 u m$.

Figure 3-19 illustrates the intensity distribution when a narrow Gaussian beam is incident into single bi-prism structure both at interface angles equal to 20 degrees. An impression of the bi-prism geometry is evident by carefully studying the figure. In Figure 3-20 the intensity distribution through the bi-prism array when a Gaussian beam of 200um beam waist propagates through 20 bi-prism EMP pixels for the same interface angles. As the deflection angle increases for the bi-prism structure, total internal reflection from the sidewalls is more obvious in this intensity distribution. 


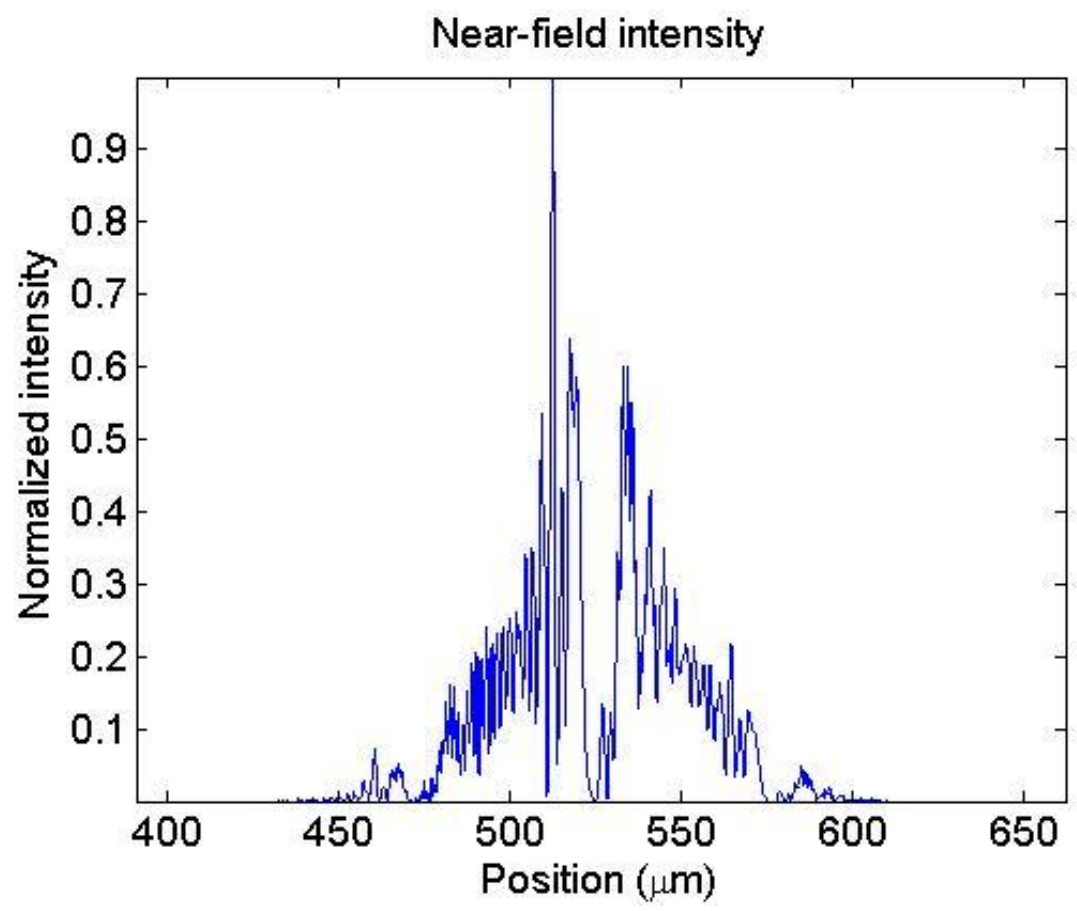

Figure 3-21: Near-field intensity for 1D bi-prism EMP arrays at $\phi_{x}=\phi_{y}=15^{\circ}$.

Far-field intensity

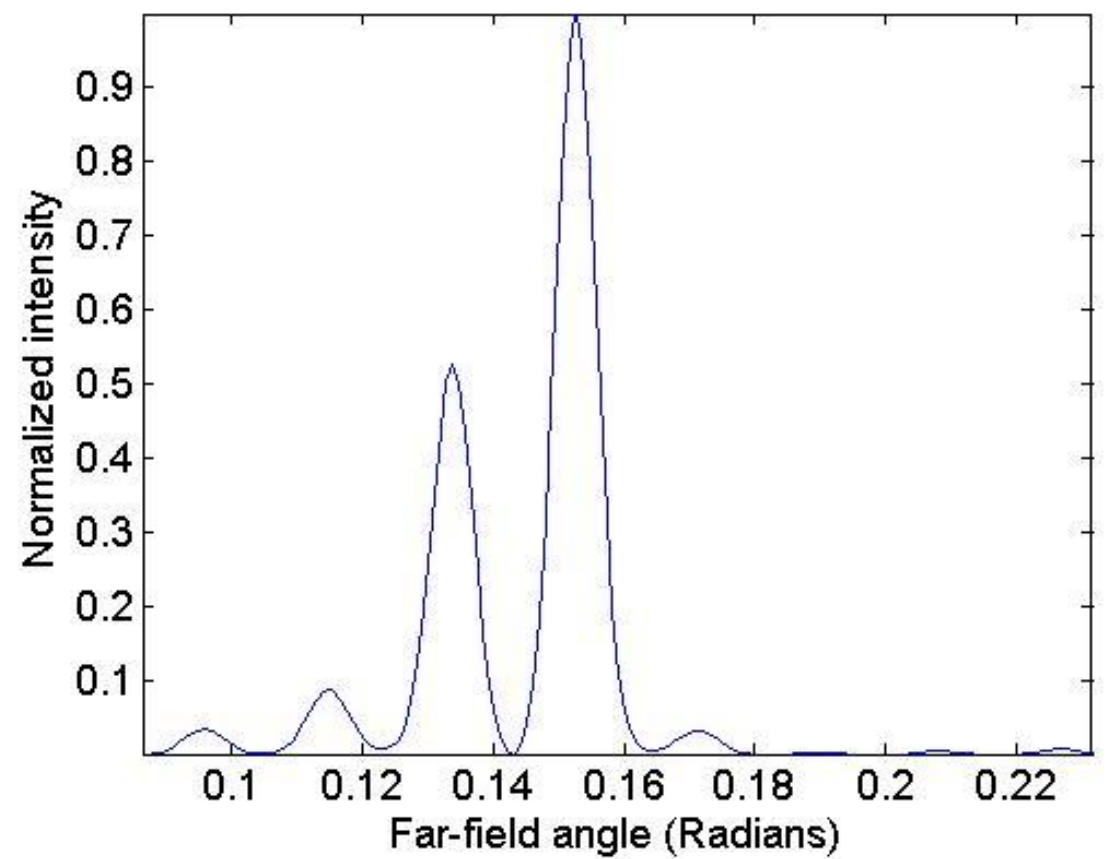

Figure 3-22: Far-field intensity for 1D bi-prism EMP arrays at $\phi_{x}=\phi_{y}=15^{\circ}$.

Figure 3-21 and Figure 3-22 are the plots of near-field and far-field intensity distribution, respectively. 3D plots with contour of far-field intensity with respect to equal 
interface angles for 1D bi-prism EMP arrays in both logarithmic and normalized scales are shown in Figure 3-23 and Figure 3-24 respectively, which summarize the beam steering efficiency results without adding a tilt angle. They are qualitatively similar to the 1D EMP results shown in Figure 3-23 and 3-24 in Chapter 3.1 except that the interface angle in bi-prism case is only up to 20 degrees while that in single-prism case ranges from 0 to 40 degrees, which gives rise to a better steering capability for bi-prism EMP arrays.

Given the fact that energy is distributed among diffraction orders, the efficiency plot for bi-prism is also discrete; the difference is that energy now is transferring at almost twice the angular rate of the single-prism case. The bi-prism design can steer to larger far-field angles using a smaller interface angle, but this is achieved at the cost of lower beam steering efficiency. A plot of efficiency versus far-field angle is shown in Figure 3-25. Phase tilt added to the bi-prism structure, also enables continuous far-field coverage as shown in Figure 3-24. 


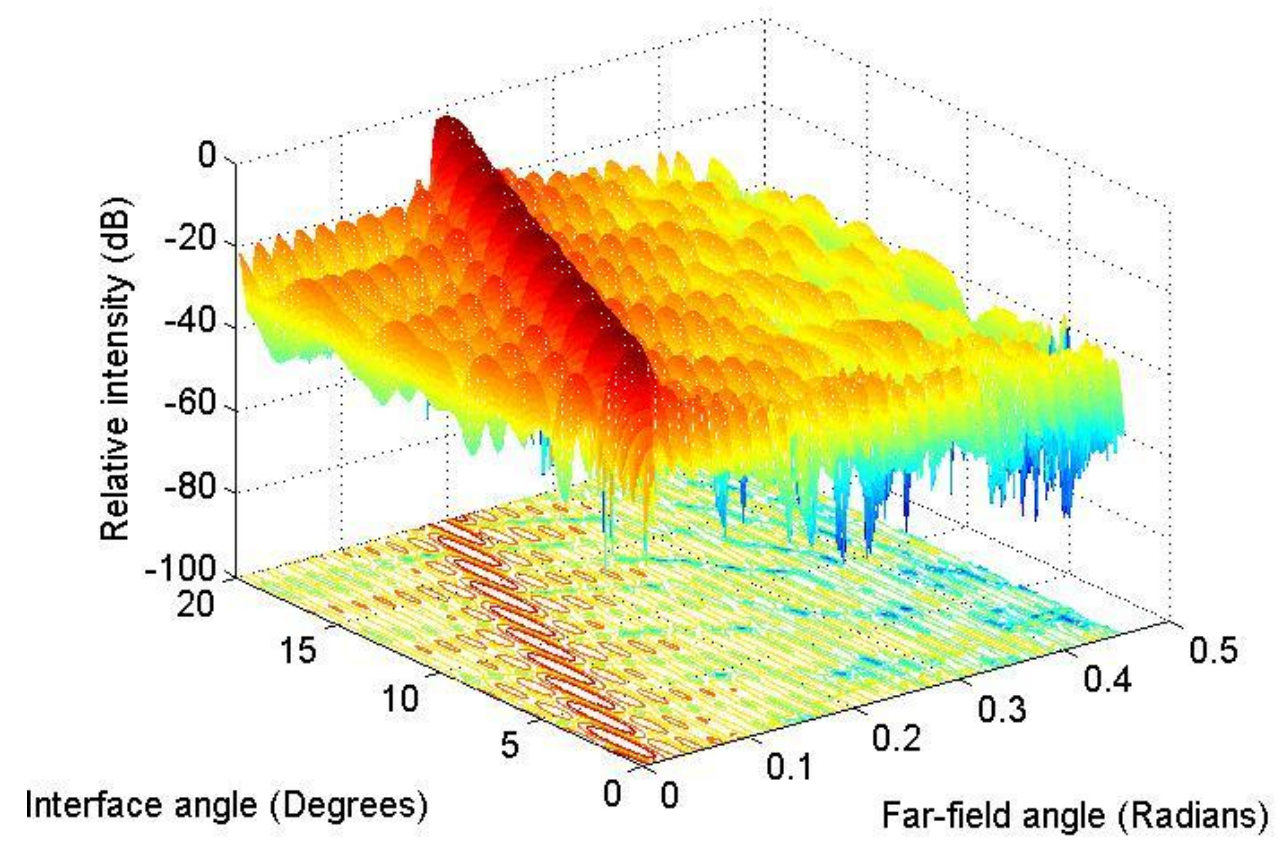

Figure 3-23: 3D mesh plot with contour of far-field intensity with respect to far-field and interface angle for 1D bi-prism EMP arrays in logarithm scale.

3D plot of 1D EMP with double prism

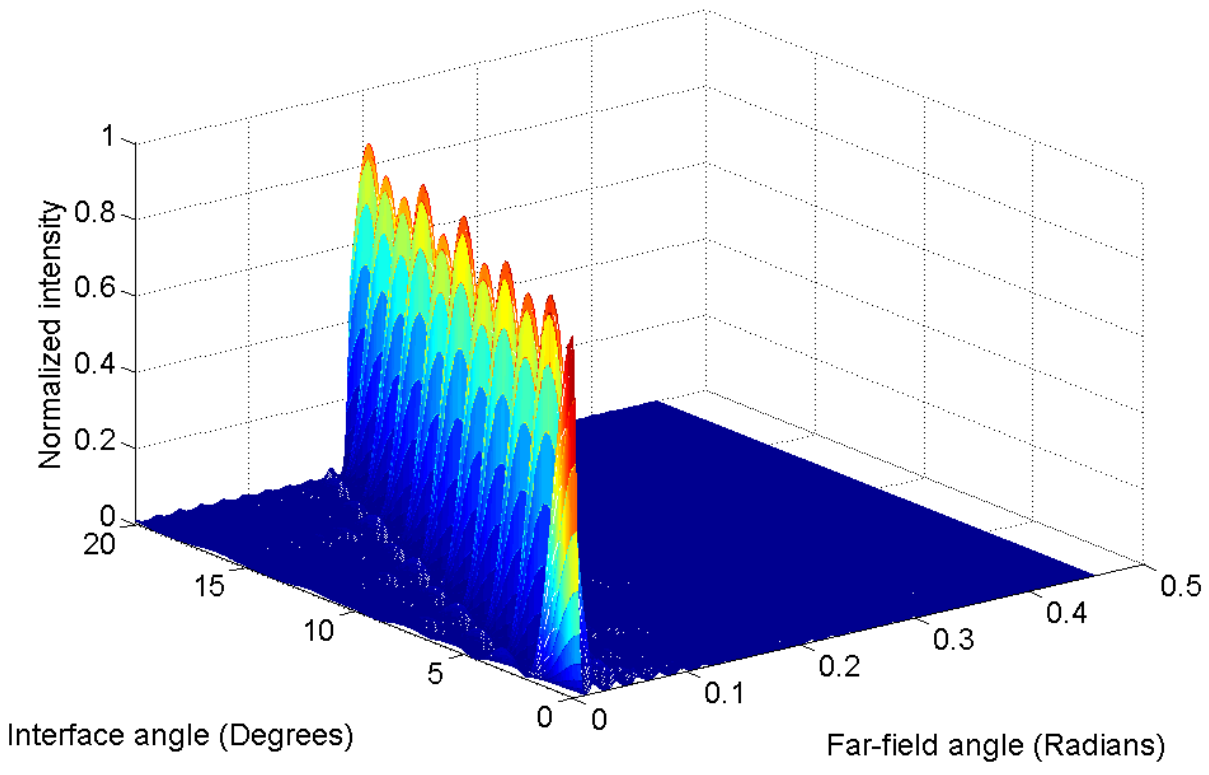

Figure 3-24: Far field angle and interface angle dependence for bi-prism EMP arrays in normalized scale. 


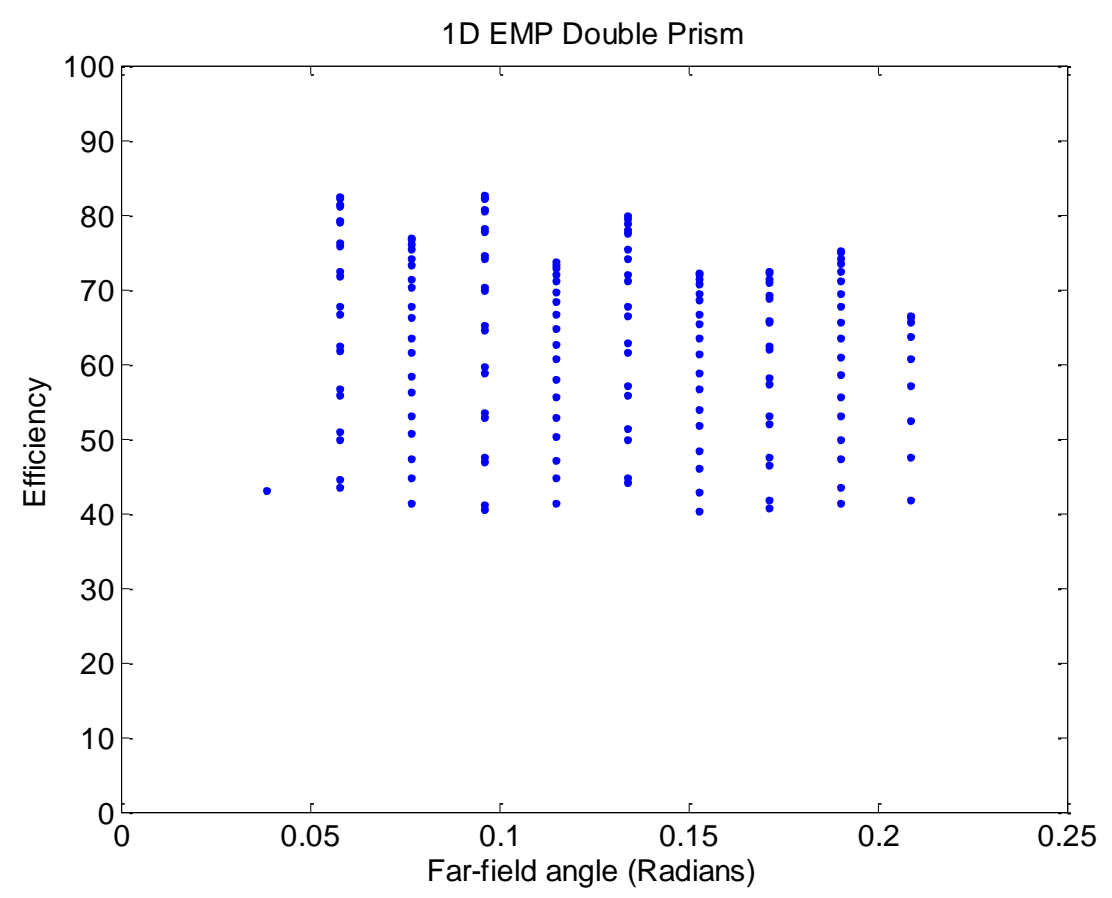

Figure 3-25: Efficiency plot with respect to far-field angle for 1D bi-prism EMP arrays.

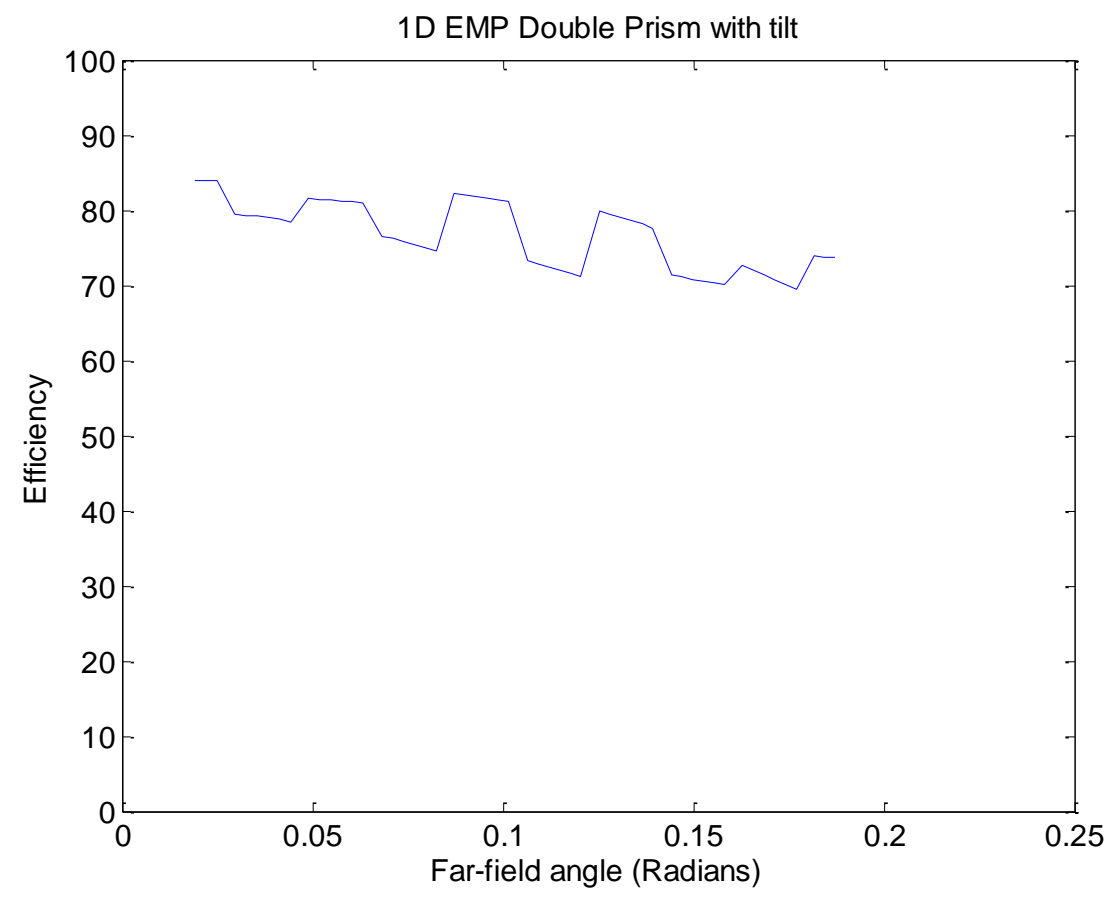

Figure 3-26: Efficiency plot of 1D bi-prism EMP arrays with phase tilt. 


\subsubsection{D BI-PRISM EMP ARRAYS}

We extended the bi-prism structure to the two-dimensional case using a geometry that is similar to the 2D prismatic arrays presented in Section 3.1. We investigated a 10x10 2D bi-prism EMP arrays in our simulation here. The two prism surfaces are mirrored and the $\mathrm{x}$ and y-direction angles are set equal (i.e. $\theta_{x}=\theta_{y}$ ). The resultant near-field and far-field intensity are shown in Figure 3-27 and Figure 3-28 respectively for interface angles equal to $15^{\circ}$. The near- and far-fields are again comparable to the 2D single-prism case discussed previously.

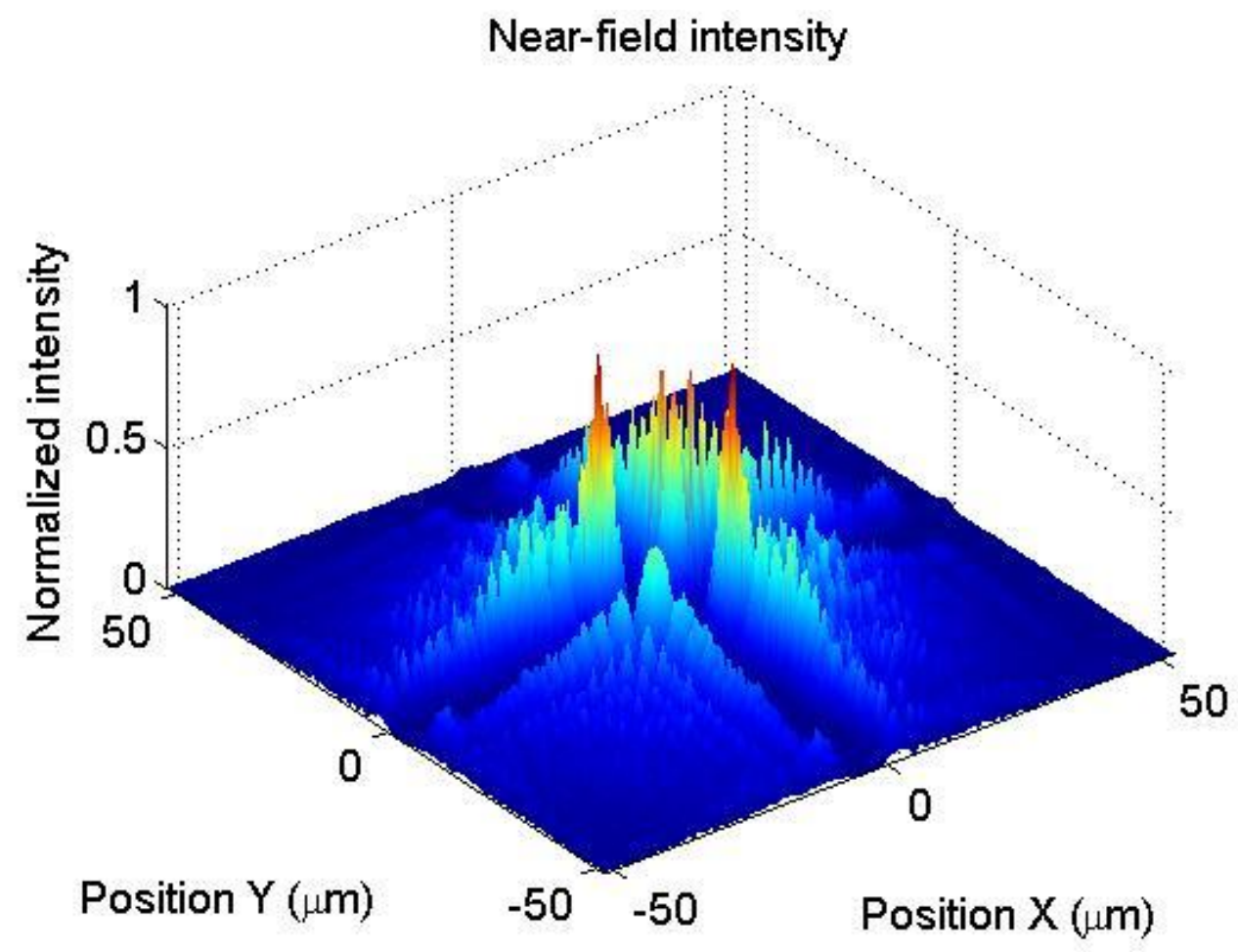

Figure 3-27: Near-field intensity of 2D bi-prism EMP arrays $\theta_{x}=\theta_{y}=15^{\circ}$. 


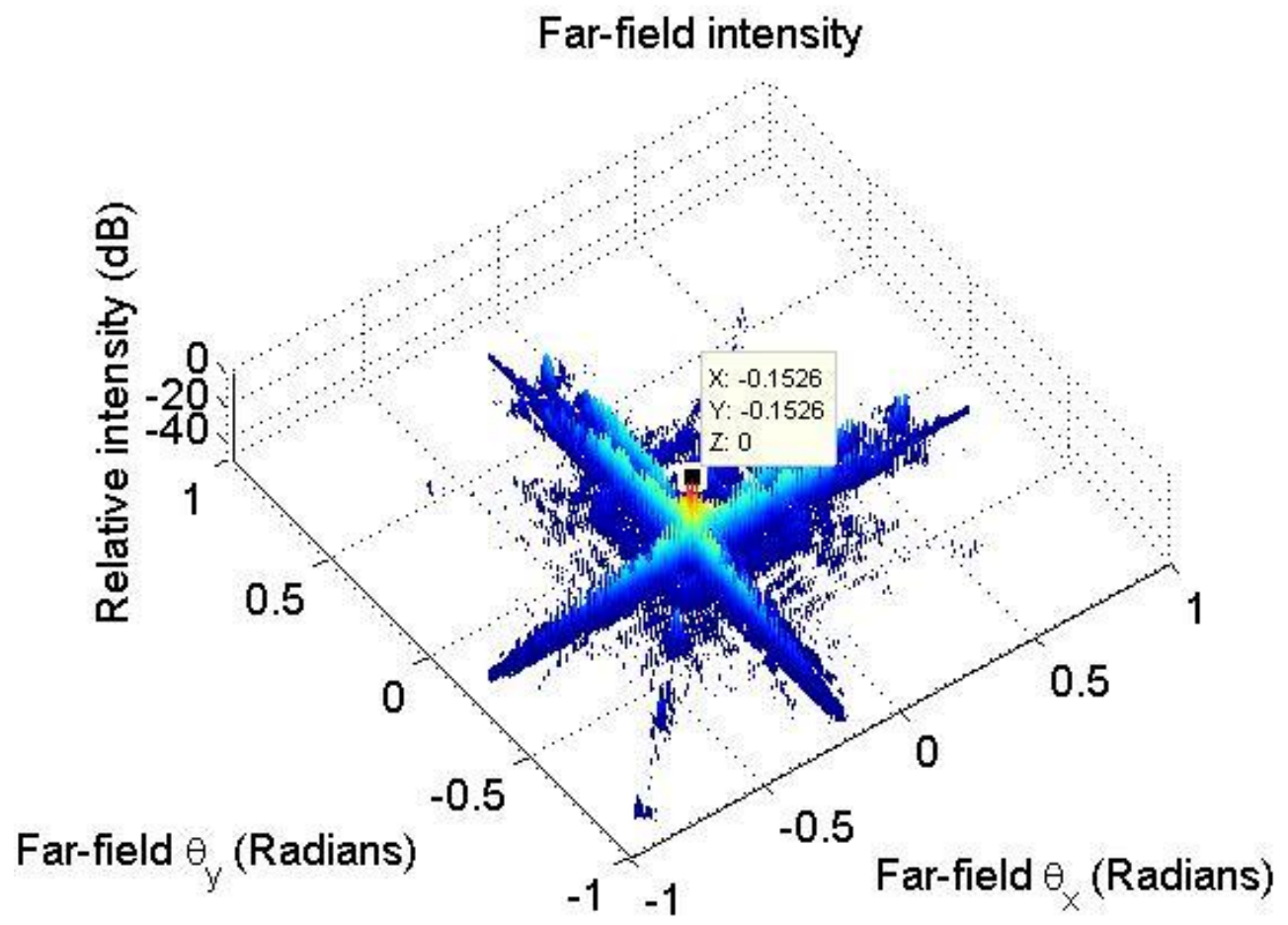

Figure 3-28: Far-field intensity of 2D bi-prism EMP arrays $\theta_{x}=\theta_{y}=15^{\circ}$.

The beam steering efficiency is evaluated using the algorithm discussed in Section 5.2 and the results are demonstrated in Figure 3-29. The spaces between discrete diffraction orders were filled using a phase tilt in Figure 3-30 which shows the continuous efficiency plot over far-field angles up to 0.2 when interface angles $\theta_{x}=\theta_{y}$ with incident beam waist of 30um. For the 2D bi-prism the efficiency drops rapidly as far-field angles increases. This geometry has a bigger loss due to the reflection at the sidewalls. 


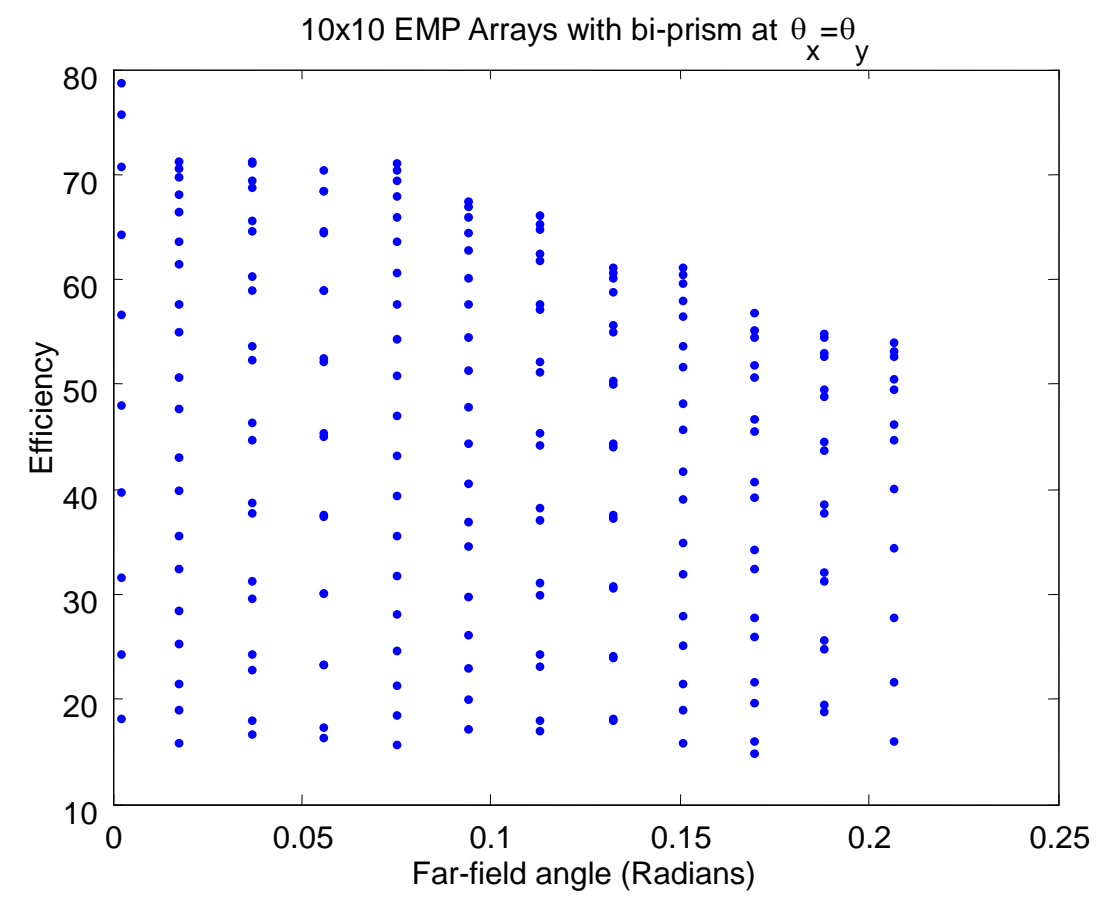

Figure 3-29: Efficiency plot with respect to far-field angles for 10x10 2D bi-prism EMP arrays.

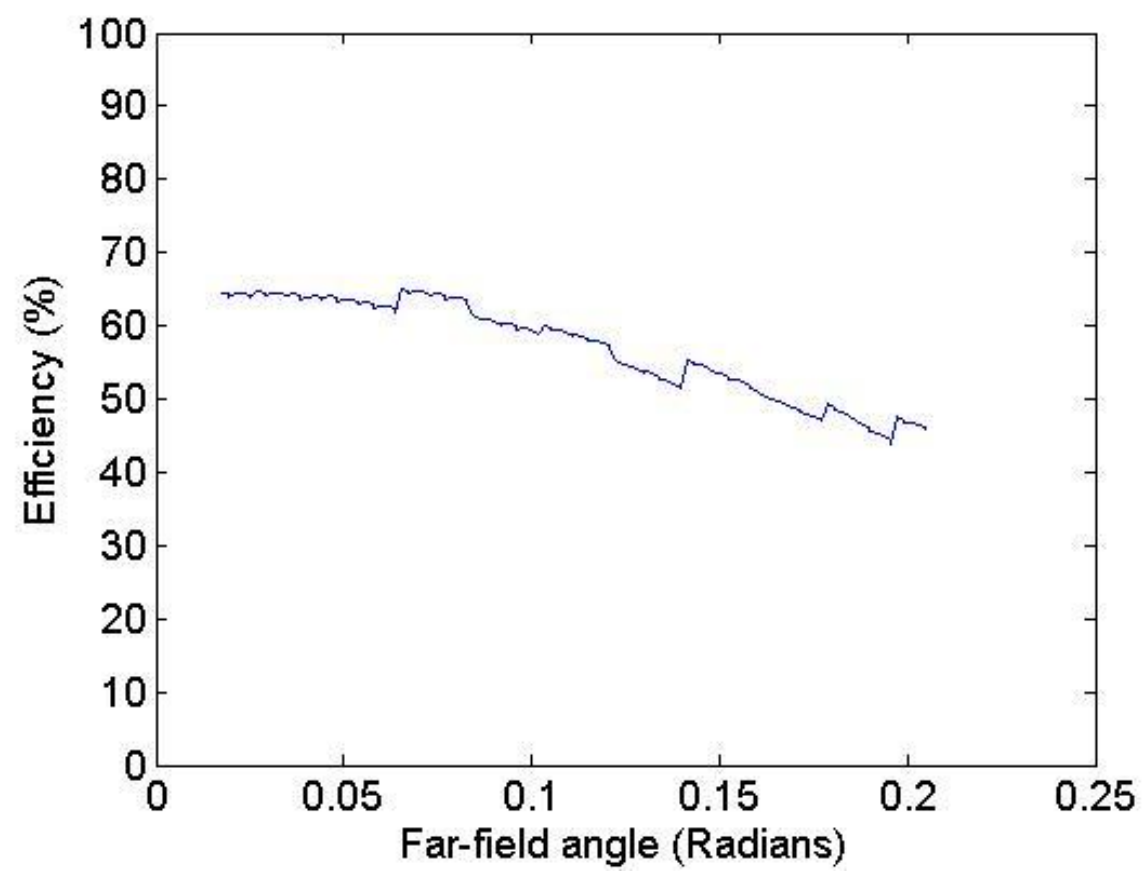

Figure 3-30: Efficiency plot of 2D bi-prism EMP arrays with phase tilt. 


\section{CONCLUSIONS}

The design of Electrowetting Microprism arrays based on standard pixellated form factors can maintain a relatively high far-field beam steering efficiency. In this thesis I studied 1D and 2D arrays of EMPs with two different pixel designs: single-prism and bi-prism geometries. The sidewalls affect the beam steering efficiency in various ways. First, light injected in them is not steered and second, they reflect light in unwanted directions.

In the $1 \mathrm{D}$ case, Beam steering of a single-prism oil/water interface design can reach to 0.2 radians at interface angle of less than $40^{\circ}$. The near-field has shallow Efficiency can be maintained above $80 \%$ for far-field angle up to angles of 0.2 radian or around 11 degrees using small tilt corrections for the phase mismatch between adjacent pixels. Our calculations are based on an extended spectral beam propagation method which is still valid for larger the off-axis diffraction angles.

Simulations of 2D single-pixel EMP arrays were limited to a 10x10 array due to memory constraints with beam width of $30 \mu \mathrm{m}$, and the far-field beam characteristics displays greater deflection of the beam. Two directions were examined steering along the $\mathrm{x}$ - (or equivalently $\mathrm{y}$-) direction, which was favorably compared with the $1 \mathrm{D}$ case to find good correspondence with the expected diffraction angle. The other direction was the 
bisecting line $(\mathrm{x}=\mathrm{y})$ when the interface is slanted along a diagonal within the box. 0.13 radians on both $\mathrm{x}$ and $\mathrm{y}$ axes can be reached in the far-field for both interface angles of $25^{\circ}$. Efficiency is found to be higher than $70 \%$ for both far-field angles of 0.1 radians. The far-field beam characteristics display lower diffraction efficiency of the beam, which can be explained by the lower fill factor in two dimensions.

Bi-prism EMP array design was also proposed and evaluated. Although this design steers to larger angles with a smaller interface angle, it comes at a cost of diffraction efficiency. In 1D case, efficiency can be maintained above $70 \%$ for far-field angle up to 0.2 radians with the help of phase tilt. In bi-prism 2D EMP arrays, efficiency further drops to $50 \%$ at both far-field angles of 0.2 radians. 


\section{FUTURE WORK}

Future work will be focused on the fabrication and experimental examination of both single-prism and bi-prism EMP arrays. A fundamentally different design without sidewalls, in other words, non-pixellated design is also of interest.

One recently proposed design is called V-COPA for liquid crystal steerers [10]. They made use of the induced geometric phase from a linearly polarized beam through a quarter wave plate, half wave plate and quarter wave plate configuration. By varying the angles between slow axis of the half wave plates and the $\mathrm{x}$ axis in laboratory coordinates, they can continuously modify the phase of linearly polarized beam by using liquid crystal modulation.

Escuti's group has developed a liquid crystal polarization grating (LCPG) beam steering device where no pixellation is used [11] [12] [13]. They used the FDTD method to simulate the performance of the LCPG introduced early in this paper [14]. With appropriate local linear polarizer patterning on the liquid crystal and carefully chosen thickness, they could eliminate the phase mismatch between sequential regions. However, the disadvantage is the minimal deflection angle step is limited by the ratio of wavelength to grating period. 
Using the design principle as an inspiration, we may be able to design a structure where micro lenses mimic the phase changes. By applying voltage to electrodes, oil and water will be physically moved and the radius of curvature of the miniature lens is changed, which will impose a phase change in far-field resulting in beam steering when the structure is in the form of an array. By removing the sidewalls, the fill factor can be improved to nearly $100 \%$ and the sidewall total reflection is also entirely eliminated. This could lead to a more efficient electrowetting beam steering structure. 


\section{BIBLIOGRAPHY}

[1] G Lippmann, "Relation entre les phenomenes electriques et capillaires," Ann. Chim. Phys., vol. 5, pp. 494-549, 1875.

[2] A Froumkine, "Couche double electrocapillarite surtension," Actualités Scientifiques, no. 373, pp. 5-36, 1936.

[3] B Berge, "Electrocapillarité et mouillage de films isolants par l'eau," C.R.A.S., no. 317, pp. 157-163, 1993.

[4] B Janocha, H Bauser, C Oehr, H Brunner, and W Gopel, "Competitive electrowetting of polymer surfaces by water and decane," Langmuir, vol. 16, pp. 3349-3354, 2000.

[5] M Vallet, M Vallade, and B Berge, "Limiting phenomena for the spreading of water on polymer films by electrowetting," The European Physical Journal B - Condensed Matter and Complex Systems, vol. 11, no. 4:583-591, 1999.

[6] Catherine Quilliet and Bruno Berge, "Electrowetting: a recent outbreak," Current Opinion in Colloid \& Interface Science, vol. 6, no. 1, pp. 34-39, February 2001.

[7] Neil R Smith, Don C Abeysinghe, Joseph W Haus, and Jason Heikenfeld, "Agile wide-angle beam steering with electrowetting microprisms," Optics Express, vol. 14, no. 14, pp. 6557-6563, July 2006.

[8] X Wang et al., "Finite-difference time-domain simulation of a liquid-crystal optical phased array," JOSA A, vol. 22, no. 2, pp. 346-354, February 2005.

[9] J Shi, P J Bos, B Winker, and P F McManamon, "Switchable optical phased prism arrays for beam steering," Proc. of SPIE, vol. 5553, p. 102-111, 2004.

[10] L Shi, P F McManamon, and P J Bos, "Liquid crystal optical phase plate with a variable in-plane gradient," Journal of Applied Physics, vol. 104, no. 3, pp. 033109-033109-7, 2008.

[11] Jihwan Kim, Chulwoo Oh, Michael J Escuti, Lance Hosting, and Steve Serati, "Wide-angle, nonmechanical beam steering using thin liquid crystal polarization gratings," Proc. of SPIE, vol. 7093, pp. 709302.1-709302.12, 2008.

[12] R K Komanduri and M J Escuti, "High efficiency reflective liquid crystal polarization gratings," Applied Physics Letters, vol. 95, no. 9, pp. 091106-091106-3, August 2009.

[13] Chulwoo Oh and Michael J Escuti, "Numerical analysis of polarization gratings using the finite-difference time-domain method," Physical Review A (Atomic, Molecular, and Optical Physics), vol. 76, no. 4, p. 043815, October 2007.

[14] Chulwoo Oh and Michael J Escuti, "Time-domain analysis of periodic anisotropic media at 
oblique incidence: an efficient FDTD implementation," Optics Express, vol. 14, no. 24, pp. 11870-11884, November 2006. 